\title{
Homogenization and numerical modelling of poroelastic materials with self-contact in the microstructure
}

\author{
Eduard Rohan, Jan Heczko \\ European Centre of Excellence, NTIS - New Technologies for Information Society, Faculty of Applied Sciences, University of West Bohemia, Univerzitní 8, 30614 Pilsen, Czech Republic
}

\section{A R T I C L E I N F O}

Article history:

Received 11 January 2019

Accepted 24 June 2019

\section{Keywords:}

Unilateral contact

Homogenization

Porous media

Variational inequality

Finite Element Method

Semi-smooth Newton method

\begin{abstract}
A B S T R A C T
We present a two-scale homogenization-based computational model of porous elastic materials subject to external loads inducing the self-contact interaction at the pore level. Microstructures under consideration are constituted as periodic lattices generated by a representative cell consisting of a solid skeleton and a pore. On its surface, the unilateral frictionless contact appears when the porous material is deformed. We focus on microstructures with rigid inclusions whereby the contact process involves opposing surfaces on the rigid and the compliant skeleton parts. A macroscopic model is derived using the periodic unfolding homogenization and the method of oscillating test functions. An efficient algorithm for the two-scale computational analysis is proposed for the numerical model obtained using the finite element discretization of the homogenized model. For this, a sequential linearization of the two-scale elasticity problem leads to the consistent effective elasticity tensor yielding consistent stiffness matrices of the macroscopic incremental formulation. The micro-level contact problem attains the form of a nonsmooth equation solved using the semi-smooth Newton method without any regularization, or problem relaxation. Numerical examples of two-dimensional deforming structures are presented as a proof of the concept. The proposed modelling approach can be extended to treat self-contact in structures subject to finite deformation.
\end{abstract}

(c) 2019 Elsevier Ltd. All rights reserved.

\section{Introduction}

The unilateral contact and self-contact problems for compliant bodies belongs to classic topics in structural mechanics. Its mathematical modeling and computational methods have been studied in a number of seminal works, see e.g. $[18,16,17]$. In the context of advanced heterogeneous materials containing different constituents distributed in the microstructure, the self-contact in the bulk may appear as the consequence of the components debonding, or cracks opening and closing, while the whole structure is being deformed. In general, the theory of the contact problems is applicable to describe behaviour of the composite and fissured materials at the microscopic level. Based on this theoretical framework, the homogenization techniques can provide an effective description of the self-contact phenomenon in the periodic, or quasi-periodic media.

The nonlinear two-scale problem treated in this paper represents an important topics in the computational homogenization which results in the so-called FEM $^{2}$ ("FEM-square") complexity of obtaining the numerical solutions using the straightforward imple-

E-mail addresses: rohan@kme.zcu.cz (E. Rohan), jheczko@ntis.zcu.cz (J. Heczko) mentation approach: at each integration point of the finite element (FE)-discretized macroscopic domain, local FE-discretized problems have to be solved to compute local microstructural responses. To alleviate computational expenses, several approaches based on the reduced-order modelling (ROM) have been proposed in recent years, including the POD (proper orthogonal decomposition) [2], or the non-uniform transformation field analysis (NUTFA), see [11] where a comparative study of different NUTFA variants was performed when employed to elastic-plasticity problems. For any homogenization-based ROM algorithm, the rigorous homogenization provides necessary relationship between increments of the thermodynamic fluxes and forces. In the context of the present paper, the effective tangential stiffness of the upscaled medium and solution algorithm to compute the local microstructure deformations and stresses in response to the macroscopic strains can be exploited to pursue the ROM computational strategy.

There is only a limited body of literature devoted to the modeling of porous materials with the self-contact interaction in the microstructure. Homogenization of the fissured media was treated in [26] using the formal approach of asymptotic expansion, leading to the homogenized macroscopic variational inequality. Further contributions to the frictionless self-contact problem with soft, or hard inclusions were handled using the two-scale 
convergence in [20], or matched asymptotic expansions [1]. In [6], the theoretical homogenization result was extended using the periodic unfolding method [5] for problems with friction and possibly rotating rigid inclusions. This work was continued in [15], where the contact between structural components distributed in thin layers was considered. Acoustic wave propagation in partially consolidated composite material containing loose particles with frictional effects was studied in [13]. In [3], the Signorini problem for the scalar elliptic operator in periodically perforated domain was considered. Therein, the derived homogenized problem is presented by a variational equation involving the "strange term" related to the negative part of the solution. The primary instability of fibre reinforced finite deforming composites in the context of self-contact and possible fibre debonding was studied in [14], whereby the bifurcation analysis was compared with numerical modelling. The computational homogenization approach to solving the contact problems was considered in [27,9]. The Arlequin method was used in [10] to solve compliant Lennard-Jones-based contact problems with adhesive instabilities.

In this paper, we follow the asymptotic analysis approach to the homogenization of the unilateral frictionless self-contact in pores of the elastic skeleton. The limit two-scale problem consists of two parts; the local problems defined for any macroscopic position within the macroscopic body are formulated in terms of the variational inequality. Its solution is driven by the local macroscopic strain tensors. Reciprocally, the local effective stress in the global problem involves solutions of the local problems. For solving such a coupled system with micro-macro transition we suggest an iterative algorithm. The state of a local configuration provides the true contact boundary which yields kinematic constraints for the linear corrector problem. Its solutions representing characteristic responses for modes of the macroscopic strain perturbations enable to define consistent tangent elasticity, such that the local contact layout determines the effective macroscopic stiffness tensors. For the discretized contact problem with strain hardening elastoplastic problem, the existence and uniqueness results were obtained in [22], whereby the formulation using the so-called nonsmooth equation was used. The same approach is pursued here to formulate the local contact problems at the microscopic level. To solve the nonsmooth equations representing a nonlinear complementarity problem (NCP), the semi-smooth Newton method proposed in [8] (cf. [4] for the smoothing conjugate gradient method) is employed which already proved to be robust and efficient when dealing with elastoplasticity in [25]. As an advantage, this method does not require any regularization, in contrast to standard solution algorithm.

The paper is organized, as follows. The self-contact problem in the porous material at the heterogeneity level (the micro-model) is established in Section 2. The asymptotic analysis of the weekformulation with respect to the scale parameter is reported in Section 3 which yields the limit two-scale problem. For its solution, an iterative scheme is proposed in Section 4, where the effective tangent stiffness is introduced and the solution algorithm is presented. In the rest of the paper, we consider microstructures with rigid inclusions which are involved in the unilateral contact with the compliant skeleton. In Section 5, the local problem defined in the representative periodic cell is modified for the contact with rigid inclusions. Also the characteristic responses yielding the homogenized stiffness are introduced. The finite element (FE) discretization of the local problems follows in Section 6, where the nonsmooth equation is derived, starting from the discretized variational inequality. Section 7 is devoted to the numerical illustration of the proposed model and the solution algorithms. Therein, we present $2 \mathrm{D}$ examples which demonstrate some features of the two-scale nonlinear computational model.
Basic notations. Through the paper we shall adhere to the following notation. The position $x$ in the medium is specified by the coordinates $\left(x_{1}, x_{2}, x_{3}\right)$ with respect to a Cartesian reference frame. We shall also use the microscopic (dilated) Cartesian reference system of coordinates $\left(y_{1}, y_{2}, y_{3}\right)$. By $\partial_{i}=\partial_{i}^{x}$ we abbreviate the partial derivative $\partial / \partial x_{i}$. We use $\nabla_{x}=\left(\partial_{i}^{x}\right)$ and $\nabla_{y}=\left(\partial_{i}^{y}\right)$ when differentiation with respect to coordinate $x$ and $y$ is applied, respectively. The symmetric gradient of a vectorial field $\boldsymbol{u}$, the strain tensor, is denoted by $\boldsymbol{e}(\boldsymbol{u})=1 / 2\left[(\nabla \boldsymbol{u})^{T}+\nabla \boldsymbol{u}\right]$, where the matrix (tensor) transpose operator is indicated by the superscript ${ }^{T}$. As usually, the vectors and tensors will be denoted by bold letters, for instance, $\boldsymbol{u}(x)$ denotes the velocity vector field depending on the spatial variable $x$. Moreover, the components of this vector will be denoted by $u_{i}$ for $i=1, \ldots, 3$, thus $\boldsymbol{u}=\left(u_{i}\right)$. The Einstein summation convention is used which stipulates implicitly that repeated indices are summed over. For any two vectors $\boldsymbol{a}, \boldsymbol{b}$, the inner product is $\boldsymbol{a} \cdot \boldsymbol{b}$. For any two 2 nd order tensors $\boldsymbol{A}, \boldsymbol{B}$ the trace of $\boldsymbol{A B}{ }^{T}$ is $\boldsymbol{A}: \boldsymbol{B}=A_{i j} B_{i j}$. By $\bar{D}$ we denote the closure of a bounded domain $D$. Further, $\boldsymbol{n}$ is the unit normal vector defined on a boundary $\partial D$, oriented outwards of $D$. In the context of an interface $\Gamma$ separating domains $\Omega_{m}$ and $\Omega_{c}$, i.e. $\Gamma=\overline{\Omega_{m}} \cap \overline{\Omega_{c}}$, normal vector $\boldsymbol{n}^{[m]}$ is outward to $\Omega_{m}$ at surface $\Gamma$. By $\mathbb{R}$ the real number set is denoted. The Lebesgue spaces of square-integrable functions on $D$ is denoted by $L^{2}(D)$, whereas $H^{1}(D)$ designates the Sobolev space $W^{1,2}(D)$ of the squareintegrable functions up to the 1 st order generalized derivative. The notation with non-bold and bold letters, i.e. like $H^{1}(D)$ and $\mathbf{H}^{1}(D)$, is used to distinguish between spaces of scalar and vector-valued functions, respectively. For any parallelepiped $Y$, such that the notion of the $Y$-periodicity can be introduced, and for any $D \subset Y, \mathbf{H}_{\#}^{1}(D)$ designates the Sobolev space $\boldsymbol{W}^{1,2}(Y)=\mathbf{H}^{1}(Y)$ of vector-valued $Y$-periodic functions (indicated by the subscript \#).

\section{Micro-model}

We consider porous elastic media constituted as periodic structures which can be generated by the so called representative periodic cells (RPC). In such a RPC, the pore geometry admits the unilateral self-contact while deforming the global structure. In this section, we introduce a micromodel describing deformation of these kind of structures whose the microstructure is characterized by the scale parameter $\varepsilon=\ell_{\text {mic }} / L$, where $\ell_{\text {mic }}$ and $L$ are the characteristic lengths of the microstructure and the macroscopic body.

\subsection{Porous structure and periodic geometry}

An open bounded domain $\Omega \subset \mathbb{R}^{d}$, with the dimension $d=2,3$, is constituted by the solid skeleton $\Omega_{s}$ and by the fractures (fissures) $\Omega_{f}$, so that

$\Omega=\Omega_{s}^{\varepsilon} \cup \Omega_{f}^{\varepsilon} \cup \Gamma^{\varepsilon}, \quad \Omega_{s}^{\varepsilon} \cap \Omega_{f}^{\varepsilon}=\varnothing, \quad \overline{\Omega_{f}^{\varepsilon}} \subset \Omega$,

where $\Gamma^{\varepsilon}=\overline{\Omega_{s}^{\varepsilon}} \cap \overline{\Omega_{f}^{\varepsilon}}$ is the interface. Further we assume that $\Omega_{s}^{\varepsilon}$ is a connected domain, whereas $\Omega_{f}^{\varepsilon}$ may not be connected. To impose boundary conditions, the decomposition of the boundary is introduced, as follows:

$$
\begin{aligned}
& \partial \Omega=\partial_{u} \Omega \cup \partial_{\sigma} \Omega, \quad \partial_{u} \Omega \cap \partial_{\sigma} \Omega=\varnothing, \\
& \Gamma_{c}^{\varepsilon}=\Gamma^{\varepsilon} \backslash \partial_{\sigma} \Omega_{s}^{\varepsilon}, \\
& \Gamma_{+}^{\varepsilon}=\Gamma_{c}^{\varepsilon} \backslash \Gamma_{-}^{\varepsilon}, \quad \Gamma_{+}^{\varepsilon} \cap \Gamma_{-}^{\varepsilon}=\varnothing,
\end{aligned}
$$

where $\partial_{\sigma} \Omega_{s}^{\varepsilon} \cap \Gamma^{\varepsilon}$ is a part of the interface $\Gamma^{\varepsilon}$ on which any contact is excluded; note that $\partial_{\sigma} \Omega \subset \partial_{\sigma} \Omega_{s}^{\varepsilon}$. Boundary $\Gamma_{c}^{\varepsilon}$ splits into two disjoint parts, such that, in the deformed configuration, points on $\Gamma_{+}^{\varepsilon}$ can get in contact with those situated on $\Gamma_{-}^{\varepsilon}$, see Fig. 1 . 

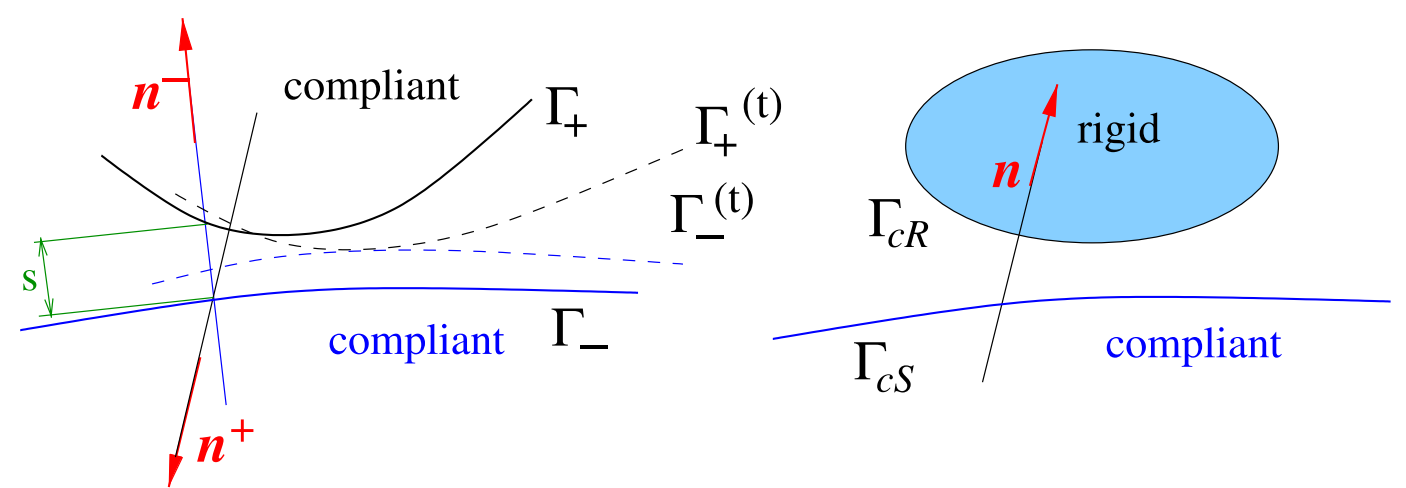

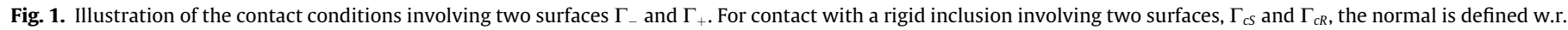
t. the rigid body.

The solid part $\Omega_{s}^{\varepsilon}$ is generated as a periodic lattice by repeating the representative volume element (RVE) occupying domain $Z^{\varepsilon}=\varepsilon Y$. The zoomed cell $\left.Y=\Pi_{i=1}^{3}\right] 0, \bar{y}_{i}\left[\subset \mathbb{R}^{3}\right.$ splits into the solid part occupying domain $Y_{s}$ and the complementary fissure part $Y_{f}$, see Fig. 2, thus

$Y=Y_{s} \cup Y_{f} \cup \Gamma^{Y}, \quad Y_{s}=Y \backslash Y_{f}, \quad \Gamma_{Y}=\overline{Y_{s}} \cap \overline{Y_{f}}$.

For a given scale $\varepsilon>0, \ell_{i}=\varepsilon \bar{y}_{i}$ is the characteristic size associated with the $i$-th coordinate direction, whereby also $\varepsilon \approx \ell_{i} / L$, hence $\ell_{i} \approx \ell^{\text {mic }}$ (for all $i=1,2,3$ ) specifies the microscopic characteristic length $\ell^{\text {mic }}$. The contact boundary is subject to the analogous split as in $(2.2)$,

$\Gamma_{c}^{Y} \subset \Gamma^{Y}, \quad \Gamma_{+}^{Y}=\Gamma_{c}^{Y} \backslash \Gamma_{-}^{Y}, \quad \Gamma_{+}^{Y} \cap \Gamma_{-}^{Y}=\varnothing$.

\subsection{Contact problem formulation}

To introduce the contact kinematic conditions, with reference to the contact boundary split (2.2) and denoting by $\boldsymbol{n}\left(x^{-}\right)$the unit normal to $\Gamma_{c}^{\varepsilon}$ at $x^{-} \in \Gamma_{-}^{\varepsilon}$, let $x^{+}=\xi \boldsymbol{n}\left(x^{-}\right)+x^{-} \in \Gamma_{+}^{\varepsilon}$ for some $\xi \geqslant 0$. Thus, two matching points on the contact surfaces $\Gamma_{+}^{\varepsilon}$ and $\Gamma_{-}^{\varepsilon}$ are introduced, which enables to define the jump []$_{n}^{\varepsilon}$ and, thereby, the contact gap function $g_{c}^{\varepsilon}$, as follows,

$$
\begin{aligned}
& g_{c}^{\varepsilon}\left(\boldsymbol{u}^{\varepsilon}\right)=\left[\boldsymbol{u}^{\varepsilon}\right]_{n}^{\varepsilon}-s^{\varepsilon}, \\
& \text { where } s^{\varepsilon}=[x]_{n}^{\varepsilon}, \\
& {[\boldsymbol{u}]_{n}^{\varepsilon}=\boldsymbol{n}\left(x^{-}\right) \cdot\left(\boldsymbol{u}\left(x^{+}\right)-\boldsymbol{u}\left(x^{-}\right)\right), \quad x^{+} \in \Gamma_{+}^{\varepsilon}, \quad x^{-} \in \Gamma_{-}^{\varepsilon} .}
\end{aligned}
$$

Thus, $s^{\varepsilon}$ is the $\varepsilon$-proportional gap clearance between the two surfaces, as measured by the two matching points. Obviously, the gap is evaluated for the normal vector which can be defined by either of the two surfaces. In Section 5, we consider microstructures with rigid inclusions which determine the normal, see Fig. 1, right. For fully compliant microstructures, alternatively a symmetric contact condition can be used such that both normals are employed, see Fig. 1, left.

We shall now introduce the friction-less contact problem for linear elastic structures subject to small strains and linearized contact conditions. Given body forces $\boldsymbol{f}^{\varepsilon}$ and external surface traction forces $\boldsymbol{b}$, find a displacement field $\boldsymbol{u}^{\varepsilon}$ which satisfies the following relationships,

$$
\begin{aligned}
& \nabla \cdot \mathbb{D} \boldsymbol{e}\left(\boldsymbol{u}^{\varepsilon}\right)+\boldsymbol{f}^{\varepsilon}=0 \quad \text { in } \Omega_{s}^{\varepsilon}, \\
& \boldsymbol{u}^{\varepsilon} \quad=0 \quad \text { on } \partial_{u} \Omega_{s}^{\varepsilon} \text {, } \\
& \boldsymbol{\sigma}^{\varepsilon} \cdot \boldsymbol{n} \quad=\boldsymbol{b}^{\varepsilon} \quad \text { on } \partial_{\sigma} \boldsymbol{\Omega}_{s}^{\varepsilon}, \\
& g_{c}^{\varepsilon}\left(\boldsymbol{u}^{\varepsilon}\right) \quad \leqslant 0 \quad \text { on } \Gamma_{c}^{\varepsilon} \text {, } \\
& \sigma_{n}^{\varepsilon} \leqslant 0 \quad \text { on } \Gamma_{c}^{\varepsilon} \text {, } \\
& g_{c}^{\varepsilon}\left(\boldsymbol{u}^{\varepsilon}\right) \sigma_{n}^{\varepsilon} \quad=0 \quad \text { on } \Gamma_{c}^{\varepsilon} \text {, } \\
& \boldsymbol{\sigma}: \boldsymbol{n} \otimes \boldsymbol{t}=0 \quad \text { on } \Gamma_{c}^{\varepsilon},
\end{aligned}
$$

where $\boldsymbol{\sigma}^{\varepsilon}=\mathbb{D} \boldsymbol{e}\left(\boldsymbol{u}^{\varepsilon}\right)$ is the stress tensor, the contact stress $\sigma_{n}^{\varepsilon}=\boldsymbol{n} \otimes \boldsymbol{n}: \boldsymbol{\sigma}^{\varepsilon}$ is the stress projection in the normal direction, and the tangent $\boldsymbol{t}$ is any unit vector satisfying $\boldsymbol{n} \cdot \boldsymbol{t}=0$.

In this paper, we shall assume vanishing traction forces on the non-contact surface of the fissures, thus, $\boldsymbol{b}^{\varepsilon} \equiv 0$ on $\partial_{\sigma} \Omega_{s}^{\varepsilon} \backslash \partial_{\sigma} \Omega$. Moreover, let $\boldsymbol{b}^{\varepsilon}=\boldsymbol{b}$ be independent of $\varepsilon$ on $\partial_{\sigma} \Omega$.

Weak solutions of the contact problem. The homogenization procedure is applied to the weak formulation of problem (2.6) which is now introduced. For this, the set of kinematically admissible displacements is needed,

$$
\mathcal{K}^{\varepsilon}=\left\{\boldsymbol{v} \in \mathbf{H}^{1}\left(\Omega_{s}^{\varepsilon}\right) \mid \boldsymbol{v}=0 \text { on } \partial_{u} \Omega_{s}^{\varepsilon}, g_{c}^{\varepsilon}(\boldsymbol{v}) \leqslant 0 \text { on } \Gamma_{c}^{\varepsilon}\right\} .
$$



Fig. 2. Periodic structure with rigid inclusions. Right: representative periodic cell $Y$. 
Definition 1. The displacement field $\boldsymbol{u}^{\varepsilon} \in \mathcal{K}^{\varepsilon}$ is a weak solution to Problem (2.6) if and only if (iff) it satisfies the variational inequality,

$$
\begin{aligned}
& \qquad \begin{aligned}
a_{\Omega}^{\varepsilon}\left(\boldsymbol{u}^{\varepsilon}, \boldsymbol{v}^{\varepsilon}-\boldsymbol{u}^{\varepsilon}\right) & \geqslant \int_{\Omega_{s}^{\varepsilon}} \boldsymbol{f}^{\varepsilon} \cdot\left(\boldsymbol{v}^{\varepsilon}-\boldsymbol{u}^{\varepsilon}\right)+\int_{\partial_{\sigma} \Omega} \boldsymbol{b} \cdot\left(\boldsymbol{v}^{\varepsilon}-\boldsymbol{u}^{\varepsilon}\right), \quad \forall \boldsymbol{v}^{\varepsilon} \in \mathcal{K}^{\varepsilon}, \\
\text { where } a_{\Omega}^{\varepsilon}(\boldsymbol{w}, \boldsymbol{v}) & =\int_{\Omega_{s}^{\varepsilon}} \operatorname{De}(\boldsymbol{w}): \boldsymbol{e}(\boldsymbol{v}) .
\end{aligned}
\end{aligned}
$$

\section{Homogenization}

The contact problem of type (2.8) has been treated by the unfolding method of homogenization in the theoretical paper [6], where a system of closed an open cracks has been considered. Since the main purpose of the present paper is to develop an effective algorithm for computing numerical solutions of the contact problem for $\varepsilon \rightarrow 0$, we present only the homogenized model arising from formulation (2.8), while the details concerning the convergence proofs are omitted here. Nevertheless, for the sake of paper completeness, we derive formally the local and global problems using the convergence results which allow us to introduce a truncated asymptotic expansion of solutions $\boldsymbol{u}^{\varepsilon}$ and to establish suitable forms of the test functions.

For the reader's convenience we recall the notion of the periodic unfolding method, see [5] for details. The periodic unfolding operator is established using the domain containing the "entire" copies of $\varepsilon Y$ only:

$$
\hat{\Omega}^{\varepsilon}=\text { interior } \bigcup_{\zeta \in \Xi^{\varepsilon}} Y_{\zeta}^{\varepsilon}, \quad Y_{\zeta}^{\varepsilon}=\varepsilon(\bar{Y}+\zeta)
$$

where $\Xi^{\varepsilon}=\left\{\zeta \in \mathbb{Z}^{3} \mid \varepsilon(\bar{Y}+\zeta) \subset \Omega\right\}$.

For all $z \in \mathbb{R}^{3}$, let $[z]$ be the unique integer such that $z-[z] \in Y$. We may write $z=[z]+\{z\}$ for all $z \in \mathbb{R}^{3}$, so that for all $\varepsilon>0$, we get the unique decomposition

$x=\varepsilon\left(\left[\frac{\chi}{\varepsilon}\right]+\left\{\frac{x}{\varepsilon}\right\}\right)=\xi+\varepsilon y \quad \forall x \in \mathbb{R}^{3}, \quad \xi=\varepsilon\left[\frac{x}{\varepsilon}\right]$.

Based on this decomposition, the periodic unfolding operator $\mathcal{T}_{\varepsilon}: L^{2}(\Omega ; \mathbb{R}) \rightarrow L^{2}(\Omega \times Y ; \mathbb{R})$ is defined as follows: for any function $v \in L^{1}(\Omega ; \mathbb{R})$, extended to $L^{1}\left(\mathbb{R}^{3} ; \mathbb{R}\right)$ by zero outside $\Omega$, i.e. $v=0$ in $\mathbb{R}^{3} \backslash \Omega$

$\mathcal{T}_{\varepsilon}(v)(x, y)=\left\{\begin{array}{cc}v\left(\varepsilon\left[\frac{\chi}{\varepsilon}\right]+\varepsilon y\right), & x \in \hat{\Omega}^{\varepsilon}, y \in Y, \\ 0 & \text { otherwise }\end{array}\right.$

Without loss of generality, in what follows, we may consider such domains $\Omega$ and such subsequences $\left\{\varepsilon_{k}\right\}$ only for which $\Omega=\hat{\Omega}^{\varepsilon_{k}}$.

For product of any $u$ and $v$ the unfolding yields $\mathcal{T}_{\varepsilon}(u v)=\mathcal{T}_{\varepsilon}(u) \mathcal{T}_{\varepsilon}(v)$. The following integration formula holds:

$\int_{\hat{\Omega}^{\varepsilon}} v d x=\frac{1}{|Y|} \int_{\Omega \times Y} \mathcal{T}_{\varepsilon}(v) d y d x \quad \forall v \in L^{1}(\Omega)$.

In what follows, for any $D \subset Y$ we abbreviate $f_{D}=\frac{1}{|Y|} \int_{D}$, Further by $\mathcal{M}_{Y}(\cdot)$ we denote the average operator over $Y$. We shall use the convergence results in the unfolded domains $\Omega \times Y$, which can be found in $[5,6]$.

The a priori estimates of $\boldsymbol{u}^{\varepsilon}$ yield the following convergence result, where the unfolding operator is employed, see [5],

$$
\begin{array}{ll}
\mathcal{T}_{\varepsilon}\left(\boldsymbol{u}^{\varepsilon}\right) & -\boldsymbol{u}^{0} \quad \text { weakly in } \mathbf{L}^{2}\left(\Omega \times Y_{S}\right), \\
\mathcal{T}_{\varepsilon}\left(\nabla \boldsymbol{u}^{\varepsilon}\right) & \rightarrow \nabla_{x} \boldsymbol{u}^{0}+\nabla_{y} \boldsymbol{u}^{1} \text { weakly in } \mathbf{L}^{2}\left(\Omega \times Y_{s}\right), \\
\frac{1}{\varepsilon}\left(\mathcal{T}_{\varepsilon}\left(\boldsymbol{u}^{\varepsilon}\right)-\mathcal{M}_{Y}^{\varepsilon}\left(\boldsymbol{u}^{\varepsilon}(x)\right)\right) & \rightarrow \nabla \boldsymbol{u}^{0} \widehat{y}+\boldsymbol{u}^{1} \quad \text { weakly in } L^{2}\left(\Omega ; \mathbf{H}^{1}\left(Y_{s}\right)\right),
\end{array}
$$

where $\boldsymbol{u}^{0} \in \mathbf{H}^{1}(\Omega)$ and $\boldsymbol{u}^{1} \in L^{2}\left(\Omega ; \mathbf{H}_{\#}^{1}\left(Y_{s}\right)\right)$, and $\widehat{y}=y-\mathcal{M}_{Y}(y)$ is the relative position with respect to the barrycenter of $Y$. Then it is straightforward to introduce the asymptotic expansion of solutions,

$\mathcal{T}_{\varepsilon}\left(\boldsymbol{u}^{\varepsilon}(x)\right)=\boldsymbol{u}^{0}(x)+\varepsilon \boldsymbol{u}^{1}(x, y)+\varepsilon^{2}(\ldots$

In analogy, we consider the truncated expansions of the test functions $\mathcal{T}_{\varepsilon}\left(\boldsymbol{v}^{\varepsilon}(x)\right)=\boldsymbol{v}^{0}(x)+\varepsilon \boldsymbol{v}^{1}(x, y), \quad$ where $\quad \boldsymbol{v}^{0} \in \mathbf{H}^{1}(\Omega) \quad$ and $\boldsymbol{v}^{1} \in L^{2}\left(\Omega ; \mathbf{H}_{\#}^{1}\left(Y_{s}\right)\right)$.

Due to properties of the unfolding operator we get $\mathcal{T}_{\varepsilon}\left(g_{c}^{\varepsilon}\left(\boldsymbol{u}^{\varepsilon}\right)\right)=\left[\mathcal{T}_{\varepsilon}\left(\boldsymbol{u}^{\varepsilon}\right)\right]_{n}^{Y}-\varepsilon[y]_{n}^{Y}$, where the jump $\left[\mathcal{T}_{\varepsilon}(\boldsymbol{v})\right]_{n}^{Y}$ is defined in analogy with $[\boldsymbol{v}]_{n}^{\varepsilon}$, see (2.5), however using traces of $\mathcal{T}_{\varepsilon}(\boldsymbol{v})$ on the matching contact surfaces $\Gamma_{ \pm}^{Y}$ of cell $Y$. Therefore, using the important convergence result (3.1) ${ }_{3}$ and the trace theorem, assuming smooth contact boundary on $\Gamma^{Y}$, one obtains

$\frac{1}{\varepsilon} \mathcal{T}_{\varepsilon}\left(g_{c}^{\varepsilon}\left(\boldsymbol{u}^{\varepsilon}\right)\right) \rightarrow\left[\nabla \boldsymbol{u}^{0} \widehat{y}+\boldsymbol{u}^{1}-\widehat{y}\right]_{n}^{Y} \quad$ weakly in $L^{2}\left(\Omega ; \mathbf{H}^{1 / 2}\left(\Gamma^{Y}\right)\right)$,

where $\hat{y}$ has been introduced by the convergence (3.1). Note that shifting the reference position by barrycenter $\mathcal{M}_{Y}(y)$ has no influence on the gap $s=[\widehat{y}]_{n}^{Y}=[y]_{n}^{Y}$ and also $\left[\nabla \boldsymbol{u}^{0} \widehat{y}\right]_{n}^{Y}=\left[\nabla \boldsymbol{u}^{0} y\right]_{n}^{Y}$. Consequently, we can introduce the gap function and the associated convex set $\mathcal{K}_{Y}$,

$$
\begin{aligned}
& \mathcal{K}_{Y}(\nabla \boldsymbol{u})=\left\{\boldsymbol{v} \in \mathbf{H}_{\#}^{1}\left(Y_{s}\right) \mid g_{c}^{Y}(\boldsymbol{v}, \nabla \boldsymbol{u}) \leqslant 0\right\}, \\
& \text { where } g_{c}^{Y}\left(\boldsymbol{u}^{1}, \nabla \boldsymbol{u}^{0}\right)=\left[\nabla \boldsymbol{u}^{0} \widehat{y}+\boldsymbol{u}^{1}-\widehat{y}\right]_{n}^{Y} .
\end{aligned}
$$

Furthermore, we shall employ the strain modes $\Pi^{i j}=\left(\Pi_{k}^{i j}\right)$ with $\Pi_{k}^{i j}=\widehat{y}_{j} \delta_{i k}$, such that, at the local level, $\Pi^{i j} e_{i j}^{\chi}\left(\boldsymbol{w}^{0}\right)$ induces the homogeneous displacement field associated to a macroscopic displacement field $\boldsymbol{w}^{0}$. Also the bilinear form related to the elasticity energy will be used,

$a_{Y_{s}}(\boldsymbol{u}, \boldsymbol{v})=f_{Y_{s}} \mathbb{D} \boldsymbol{e}_{y}(\boldsymbol{u}): \boldsymbol{e}_{y}(\boldsymbol{v}), \quad$ where $f_{Y_{s}}(\mathrm{l})=\frac{1}{|Y|} \int_{Y_{s}}()$.

With this notation in hand, the limit two-scale contact problem can be defined.

Definition 2. For given $\overline{\boldsymbol{f}} \in \mathbf{L}^{2}(\Omega)$ and $\boldsymbol{b} \in \mathbf{L}^{2}\left(\partial_{\sigma} \Omega\right)$, find a two-scale solution $\left(\boldsymbol{u}^{0}, \boldsymbol{u}^{1}\right)$, such that the Local and the Global equilibria hold:

Local equilibrium: for a.a. $x \in \Omega$, the fluctuating displacement fields $\boldsymbol{u}^{1}(x, \cdot) \in \mathcal{K}_{Y}\left(\nabla \boldsymbol{u}^{0}\right)$, satisfy

$$
a_{Y_{s}}\left(\boldsymbol{u}^{1}+\Pi^{i j} e_{i j}^{\chi}\left(\boldsymbol{u}^{0}\right), \boldsymbol{v}-\boldsymbol{u}^{1}\right) \geqslant 0, \quad \forall \boldsymbol{v} \in \mathcal{K}_{Y}\left(\nabla \boldsymbol{u}^{0}\right) .
$$

Global equilibrium: Macroscopic displacement $\boldsymbol{u}^{0} \in U_{0}(\Omega)$, where $U_{0}(\Omega)=\left\{\boldsymbol{v} \in \mathbf{H}^{1}(\Omega) \mid \boldsymbol{v}=0\right.$ on $\left.\partial_{u} \Omega\right\}$, satisfies

$$
\begin{aligned}
& \int_{\Omega} \boldsymbol{\sigma}^{0}\left(\boldsymbol{u}^{0}, \boldsymbol{u}^{1}\right): \boldsymbol{e}_{x}\left(\boldsymbol{v}^{0}\right)=\int_{\Omega} \overline{\boldsymbol{f}} \cdot \boldsymbol{v}^{0}+\int_{\partial_{\sigma} \Omega} \boldsymbol{b} \cdot \boldsymbol{v}^{0} \quad \forall \boldsymbol{v} \in U_{0}(\Omega), \\
& \text { with } \boldsymbol{\sigma}^{0}=\left(\sigma_{i j}^{0}\right), \quad \sigma_{i j}^{0}=a_{Y_{s}}\left(\boldsymbol{u}^{1}+\Pi^{k l} e_{k l}^{x}\left(\boldsymbol{u}^{0}\right), \Pi^{i j}\right) .
\end{aligned}
$$

The volume forces $\overline{\boldsymbol{f}}$ in the limit problem are related to $\boldsymbol{f}^{\varepsilon}$ involved in problem (2.8). In the simplest case, $\boldsymbol{f}^{\varepsilon}=\boldsymbol{f}$ is a constant vector, so that $\overline{\boldsymbol{f}}=\phi_{\mathrm{s}} \boldsymbol{f}$ with $\phi_{s}=\left|Y_{s}\right| /|Y|$. In general, we assume existence of $\overline{\boldsymbol{f}}=\mathcal{f}_{Y_{s}} \tilde{\boldsymbol{f}}$ whereby $\mathcal{T}_{\varepsilon}\left(\boldsymbol{f}^{\varepsilon}\right)-\tilde{\boldsymbol{f}}$ weakly in $\mathbf{L}^{2}\left(\Omega \times Y_{s}\right)$. 
The following proposition summarizes the results of [6] interpreted for boundary conditions which guarantee coerciveness of the bilinear form $a_{\Omega}^{\varepsilon}(\cdot, \cdot)$ with respect to set $\mathcal{K}^{\varepsilon}$ independently of $\varepsilon$.

Proposition 1. Solutions $\boldsymbol{u}^{\varepsilon}$ of problem (2.8) converge in the sense of (3.1) to the two-scale solutions $\mathcal{S}:=\left(\boldsymbol{u}^{0}, \boldsymbol{u}^{1}\right)$ of the limit problem (3.6) and (3.7).

Proof of Proposition 1. The constructive part of the proof consists in substituting the truncated expansion (3.2) in the variational inequality (2.8). By virtue of the unfolding integration formula and due to the convergence (3.1), one obtains

$$
\begin{aligned}
& \int_{\Omega} f_{Y_{s}} \mathbb{D}\left(\boldsymbol{e}_{y}\left(\boldsymbol{u}^{1}\right)+\boldsymbol{e}_{x}\left(\boldsymbol{u}^{0}\right)\right):\left(\boldsymbol{e}_{y}\left(\boldsymbol{v}^{1}\right)+\boldsymbol{e}_{x}\left(\boldsymbol{v}^{0}\right)-\boldsymbol{e}_{y}\left(\boldsymbol{u}^{1}\right)-\boldsymbol{e}_{x}\left(\boldsymbol{u}^{0}\right)\right) \\
& \geq \int_{\Omega} \overline{\boldsymbol{f}} \cdot\left(\boldsymbol{v}^{0}-\boldsymbol{u}^{0}\right)+\int_{\partial_{\sigma} \Omega} \boldsymbol{b} \cdot\left(\boldsymbol{v}^{0}-\boldsymbol{u}^{0}\right),
\end{aligned}
$$

which has to be satisfied for test displacements $\left(\boldsymbol{v}^{1}, \boldsymbol{v}^{0}\right)$ subject to the kinematic constraints. To specify them, we need to determine the limit set of kinematically admissible displacements. By virtue of the convergence (3.3), for any $\boldsymbol{v}^{\varepsilon} \in \mathcal{K}^{\varepsilon}$ and the limit functions $\boldsymbol{v}^{0}$ and $\boldsymbol{v}^{1}$ satisfying (3.1), as interpreted for $\boldsymbol{v}^{\varepsilon}$, one obtains

$g_{c}^{Y}\left(\boldsymbol{v}^{1}, \nabla \boldsymbol{v}^{0}\right)=\left[\nabla \boldsymbol{v}^{0} \widehat{y}+\boldsymbol{v}^{1}-\widehat{y}\right]_{n}^{Y} \leqslant 0$.

Obviously, the same inequality must be satisfied by the solution pair, thus $g_{c}^{Y}\left(\boldsymbol{u}^{1}, \nabla \boldsymbol{u}^{0}\right) \leqslant 0$. Inequality (3.8) has to be satisfied for any pair $\left(\boldsymbol{v}^{1}, \boldsymbol{v}^{0}\right)$ of test functions satisfying the contact kinematic condition (3.9). In the homogenization procedure, to distinguish the local and global problems, the limit two-scale relationship (i.e. (3.8) in our case) has to be tested by suitable test functions. Usually, the local problems are obtained for vanishing macroscopic test functions. On the contrary, the testing by the macroscopic functions while letting vanish two-scale functions yields the global problem. In a special case of the zero clearance (closed cracks), i.e. $\hat{s}_{0}:=[\widehat{y}]_{n}^{Y}=0$, the gradient $\nabla \boldsymbol{v}^{0}$ does not influence the gap function. To cope with positive clearances $\hat{s}_{0} \geqslant 0$, we may consider suitable combinations of the test functions. By introducing $\hat{\boldsymbol{w}}\left(\tilde{\boldsymbol{v}}^{0}\right) \in L^{2}\left(\Omega ; \mathbf{H}_{\#}^{1}(Y)\right)$ such that $\hat{\boldsymbol{w}}\left(\tilde{\boldsymbol{v}}^{0}\right)=\nabla_{x} \tilde{\boldsymbol{v}}^{0} \hat{y}$ on $\Gamma_{c}^{Y}$ for $\tilde{\boldsymbol{v}}^{0} \in \mathbf{H}^{1}(\Omega)$, the local test functions compensate effects of the gap dilation caused by affine transformation induced by $\nabla_{x} \tilde{\boldsymbol{v}}^{0}$. Let $\tilde{\boldsymbol{v}}^{1} \in L^{2}\left(\Omega ; \mathbf{H}_{\#}^{1}(Y)\right)$ and introduce

$\boldsymbol{v}^{1}:=\tilde{\boldsymbol{v}}^{1}-\hat{\boldsymbol{w}}\left(\tilde{\boldsymbol{v}}^{0}\right), \quad$ while $\hat{\boldsymbol{w}}\left(\tilde{\boldsymbol{v}}^{0}\right)=\nabla_{x} \tilde{\boldsymbol{v}}^{0} \widehat{y}$,

$\boldsymbol{v}^{0}:=\boldsymbol{u}^{0}+\tilde{\boldsymbol{v}}^{0}$

Clearly, for such a selection of the test functions we get

$$
\begin{aligned}
g_{c}^{Y}\left(\boldsymbol{v}^{1}, \nabla \boldsymbol{v}^{0}\right) & =\left[\nabla_{x}\left(\tilde{\boldsymbol{v}}^{0}+\boldsymbol{u}^{0}\right) \widehat{y}+\tilde{\boldsymbol{v}}^{1}-\hat{\boldsymbol{w}}\left(\tilde{\boldsymbol{v}}^{0}\right)-\widehat{y}\right]_{n}^{Y} \\
& =\left[\nabla_{x} \boldsymbol{u}^{0} \widehat{y}+\tilde{\boldsymbol{v}}^{1}-\hat{y}\right]_{n}^{Y},
\end{aligned}
$$

so that $\tilde{\boldsymbol{v}}^{0}$ is not constrained by the non-penetration condition (3.9). Now, using the expressions (3.7) $)_{2}$ for $\boldsymbol{\sigma}^{0}$, and using (3.10), from (3.8) we get the following inequality to be satisfied by pair $\left(\boldsymbol{u}^{0}, \boldsymbol{u}^{1}\right)$, such that $\boldsymbol{u}^{1} \in \mathcal{K}_{Y}\left(\nabla \boldsymbol{u}^{0}\right)$ and $\boldsymbol{u}^{0} \in U_{0}(\Omega)$,

$$
\begin{aligned}
& \int_{\Omega} a_{Y_{s}}\left(\boldsymbol{u}^{1}+\Pi^{k l} e_{k l}^{x}\left(\boldsymbol{u}^{0}\right), \tilde{\boldsymbol{v}}^{1}-\hat{\boldsymbol{w}}\left(\tilde{\boldsymbol{v}}^{0}\right)-\boldsymbol{u}^{1}\right) \\
& +\int_{\Omega} \boldsymbol{\sigma}^{0}\left(\boldsymbol{u}^{0}, \boldsymbol{u}^{1}\right): \boldsymbol{e}_{x}\left(\tilde{\boldsymbol{v}}^{0}\right) \geqslant \int_{\Omega} \overline{\boldsymbol{f}} \cdot \tilde{\boldsymbol{v}}^{0}+\int_{\partial_{\sigma} \Omega} \boldsymbol{b} \cdot \tilde{\boldsymbol{v}}^{0},
\end{aligned}
$$

for all $\left(\tilde{\boldsymbol{v}}^{1}, \tilde{\boldsymbol{v}}^{0}\right)$, such that $\tilde{\boldsymbol{v}}^{0} \in U_{0}(\Omega), \tilde{\boldsymbol{v}}^{1} \in \hat{\boldsymbol{w}}\left(\tilde{\boldsymbol{v}}^{0}\right)+\mathcal{K}_{Y}\left(\nabla \boldsymbol{u}^{0}\right)$.

By taking $\tilde{\boldsymbol{v}}^{0} \equiv 0$, (3.12) yields problem (3.6). Since this inequality has to be satisfied for any $\boldsymbol{v}^{1} \in \mathcal{K}_{Y}\left(\nabla \boldsymbol{u}^{0}\right)$, thus also for $\hat{\boldsymbol{w}}\left(\tilde{\boldsymbol{v}}^{0}\right) \in \mathcal{K}_{Y}\left(\nabla \boldsymbol{u}^{0}\right)$, inequality (3.12) is satisfied also for any $\tilde{\boldsymbol{v}}^{0} \in U_{0}(\Omega)$, if the following identity holds,

$\int_{\Omega} \boldsymbol{\sigma}^{0}\left(\boldsymbol{u}^{0}, \boldsymbol{u}^{1}\right): \boldsymbol{e}_{x}\left(\tilde{\boldsymbol{v}}^{0}\right)=\int_{\Omega} \overline{\boldsymbol{f}} \cdot \tilde{\boldsymbol{v}}^{0}+\int_{\partial_{\sigma} \Omega} \boldsymbol{b} \cdot \tilde{\boldsymbol{v}}^{0}$.

Hence, we conclude that the limit solutions of (2.8) must satisfy the coupled problems (3.6) and (3.7). $\square$

\section{Iterative solution algorithms}

Problem introduced in Definition 1 is constituted by two strongly coupled and nonlinear subproblems (3.6) and (3.7). For a linear system of partial differential equations (PDEs), it is possible to introduce characteristic solutions of the local cell problems and, consequently, to compute the homogenized coefficients involved in the macroscopic problem. However, in general, such a treatment is not possible when dealing with nonlinear problems.

The commonly accepted numerical approach to solving multiscale nonlinear problems is based on the so-called "FE-square" strategy. Local problems are solved using FEM (although any convenient numerical method can be used) whereby an approximation of the macroscopic structural response presents an input. In this way, effective medium coefficients (such as elasticity) can be established for all microstructures distributed in the macroscopic domain (e.g. at each finite element). This enables to establish the macroscopic problem which can be solved to compute next approximation of the macroscopic response. That is why a linearization scheme with micro-macro transitions is introduced. To obtain a convergent sequence of the two scale approximate solutions, the linearization must be consistent in the sense which is explained below.

\subsection{Sequential consistent linearization}

This approach is based upon decompositions of the total deformation (displacements and strains) into incremental steps: these can be viewed as corrections to the previous trial steps, or (more appropriately) as increments associated with load steps applied in time (forces or prescribed displacements on a boundary segment). Such incremental formulations allow for a consistent linearization with respect to an infinitesimal perturbation.

Time increments and updated configuration. Although the contact problem is time-independent, we can introduce a pseudo-time $t$ which is considered as an applied load parameter. Here we explain the construction of the map $\mathcal{F}:\left(G, x, \mathcal{S}^{(t)}\right) \mapsto G^{(t)}$, where $G$ is a manifold or a subdomain $G \subset Y, x \in \Omega$ in the reference configuration (for $t=0$ ), and $\mathcal{S}^{(t)}=\left(\boldsymbol{u}^{0,(t)}, \boldsymbol{u}^{1,(t)}\right)$ is the current two-scale state. Let indices $(t)$ and $(t+\Delta t)$ label two consecutive configurations, i.e. we use $\Omega^{(t)}$ and (for $x \in \Omega^{(t)}$ ) the micro configurations $Y^{(t)}(x)$. Consequently, the total displacements can be expressed in terms of increments $\left(\Delta \boldsymbol{u}^{0}(x), \Delta \boldsymbol{u}^{1}(x, y)\right)$ defined in the current macroscopic configuration $\Omega^{(t)}$ and local configurations $Y^{(t)}(x)$, for $x \in \Omega^{(t)}$.

$$
\begin{aligned}
\boldsymbol{u}^{0,(t+\Delta t)}(x) & =\boldsymbol{u}^{0,(t)}(x)+\Delta \boldsymbol{u}^{0}(x), \\
\boldsymbol{u}^{1,(t+\Delta t)}(x, y) & =\boldsymbol{u}^{1,(t)}(x, y)+\Delta \boldsymbol{u}^{1}(x, y) .
\end{aligned}
$$

By virtue of the micro-displacements,

$$
\begin{aligned}
\boldsymbol{u}^{\mathrm{mic},(t)}(x, y) & =\boldsymbol{u}^{1,(t)}(x, y)+\nabla_{x} \boldsymbol{u}^{0,(t)}(x) \widehat{y}, \quad x \in \Omega, \quad y \in Y, \\
\text { or } \Delta \boldsymbol{u}^{\mathrm{mic}}(x, y) & =\Delta \boldsymbol{u}^{1}(x, y)+\nabla_{x} \Delta \boldsymbol{u}^{0}(x) \widehat{y}, \quad x \in \Omega^{(t)}, \quad y \in Y^{(t)}(x),
\end{aligned}
$$


the updating scheme can be introduced, as follows,

$$
\begin{aligned}
& x^{\prime}:=x+\Delta \boldsymbol{u}^{0}(x) \quad x \in \Omega^{(t)}, \\
& y^{\prime}:=y+\Delta \boldsymbol{u}^{\mathrm{mic}}(x, y) \quad x \in \Omega^{(t)}, \quad y \in Y^{(t)}(x), \\
& \Omega^{(t+\Delta t)}=\Omega^{(t)}+\left\{\Delta \boldsymbol{u}^{0}(x)\right\}_{x \in \Omega^{(t)}}, \\
& Y^{(t+\Delta t)}\left(x^{\prime}\right)=Y^{(t)}(x)+\left\{\Delta \boldsymbol{u}^{\mathrm{mic}}(x, y)\right\}_{x \in \Omega^{(t)}, y \in Y^{(t)}(x)},
\end{aligned}
$$

where $\Omega+\{f(x)\}_{x \in \Omega}$ is defined as the point-wise additive operation. Since the updating scheme 4.1, 4.2 and 4.3 is linear, it holds that $\mathcal{F}\left(Y, x, \mathcal{S}^{(t+\Delta t)}\right) \equiv \mathcal{F}\left(Y^{(t)}, x^{(t)}, \Delta \mathcal{S}^{(t)}\right)$, where $x^{(t)} \in \Omega^{(t)}$ is the spatial position of $x \in \Omega^{(0)}$ and the two-scale state increment is $\Delta \mathcal{S}^{(t)}=\left(\Delta \boldsymbol{u}^{0}, \Delta \boldsymbol{u}^{1}\right)$ is the one employed in (4.3).

Tangent elastic stiffness. Let $\Gamma_{*}^{(t)}(x)=\Gamma_{+}^{(t)}(x) \cap \Gamma_{-}^{(t)}(x)$ be the current "true contact surface" of the updated configuration at $(t)$, where $x \in \Omega^{(t)}$. The two matching contact surfaces coincide at the current deformed configuration.

We consider a small macroscopic perturbation $\delta \boldsymbol{u}^{0}$ with respect to the state at time $t$, i.e. configuration labelled by ${ }^{(t)}$, such that the contact distribution does not change. This is due to fixed sets of active and inactive constraints (a simplification which disregards semiactive constraint sets requiring the B-differentiability, see e.g.[21] and the construction of Clark's sub-differentials [7]).

For a given displacement field $\boldsymbol{U} \in \mathbf{H}^{1}\left(Y^{(t)}\right)$, let us introduce the set of admissible displacement perturbations,

$V_{0}\left(\boldsymbol{U}, Y_{s}^{(t)}, x\right)=\left\{\boldsymbol{v} \in \mathbf{H}_{\#}^{1}\left(Y_{s}^{(t)}\right) \mid[\boldsymbol{v}+\boldsymbol{U}]_{n}^{(t)}=0\right.$ on $\left.\Gamma_{*}^{(t)}(x)\right\}$.

To compute a local perturbation $\delta \boldsymbol{u}^{1}$ of the affine map induced by $\delta \boldsymbol{u}^{0}$, we define $\delta \boldsymbol{U}^{0}=\Pi^{i j} e_{i j}^{x}\left(\delta \boldsymbol{u}^{0}\right)$ and find $\delta \boldsymbol{u}^{1} \in V_{0}\left(\delta \boldsymbol{U}^{0}, Y_{s}^{(t)}, x\right)$ satisfying the linear equation,

$f_{Y_{s}} \mathbb{D} \boldsymbol{e}_{y}\left(\delta \boldsymbol{u}^{1}+\delta \boldsymbol{U}^{0}\right): \boldsymbol{e}_{y}(\boldsymbol{v})=0, \quad \forall \boldsymbol{v} \in V_{0}\left(0, Y_{s}, x\right)$.

The definition of set $V_{0}\left(\boldsymbol{U}, Y_{s}^{(t)}, x\right)$ reflects the true contact set $\Gamma_{*}$, see also Remark 1 presented below in the context of an iterative algorithm.

By virtue of the linearity (due to the bilateral contact and having fixed the active contact set $\left.\Gamma_{*}^{(t)}\right)$, the so-called corrector problem for the decomposition $\delta \boldsymbol{u}^{1}(x, y)=\boldsymbol{w}^{i j}(x, y) e_{i j}^{x}\left(\delta \boldsymbol{u}^{0}\right)$ can be introduced, where $\boldsymbol{w}^{i j}(x, \cdot) \in V_{0}\left(\boldsymbol{\Pi}^{i j}, Y_{s}^{(t)}, x\right)$ satisfies

$f_{Y_{s}^{(t)}} \mathbb{D} \boldsymbol{e}_{y}\left(\mathbf{w}^{i j}+\Pi^{i j}\right): \boldsymbol{e}_{y}(\boldsymbol{v})=0, \quad \forall \boldsymbol{v} \in V_{0}\left(0, Y_{s}^{(t)}, x\right)$.

Then the effective tangent modulus $\mathbb{D}^{H,(t)}=\left(D_{i j k l}^{H,(t)}\right)$ is computed with its components:

$D_{i j k l}^{H,(t)}=\mathcal{f}_{Y_{s}^{(t)}} \mathbb{D} \boldsymbol{e}_{y}\left(\boldsymbol{w}^{k l}+\boldsymbol{\Pi}^{k l}\right): \boldsymbol{e}_{y}\left(\boldsymbol{w}^{i j}+\boldsymbol{\Pi}^{i j}\right)$.

It is easy to see that the effective stress perturbation $\delta \boldsymbol{\sigma}^{0}$ is expressed using (4.2) and (4.7), indeed

$$
\begin{aligned}
\delta \sigma^{0} & =\oint_{Y_{s}^{(t)}} \mathbb{D} \boldsymbol{e}_{y}\left(\delta \boldsymbol{u}^{\mathrm{mic}}\right)=\oint_{Y_{s}^{(t)}} \mathbb{D} \boldsymbol{e}_{y}\left(\delta \boldsymbol{u}^{1}+\boldsymbol{\Pi}^{k l} e_{k l}^{x}\left(\delta \boldsymbol{u}^{0}\right)\right) \\
& =\oint_{Y_{s}^{(t)}} \mathbb{D} \boldsymbol{e}_{y}\left(\boldsymbol{w}^{k l}+\boldsymbol{\Pi}^{k l}\right) e_{k l}^{x}\left(\delta \boldsymbol{u}^{0}\right) \\
& =\mathbb{D}^{H,(t)} \boldsymbol{e}_{x}\left(\delta \boldsymbol{u}^{0}\right) .
\end{aligned}
$$

\subsection{Incremental formulation and solution algorithm}

We present an algorithm to compute the new time level at $t+\Delta t$ for a given configuration at $t$. The algorithm constructs a sequence of iterations $0 \leqslant i, i+1, \ldots$ to approximate $\boldsymbol{u}^{0,(t+\Delta t)}(x)$ and $\boldsymbol{u}^{1,(t+\Delta t)}(x, y)$. Thereby, approximations of $\Omega^{i} \approx \Omega^{(t+\Delta t)}$ and $Y^{i} \approx Y^{(t+\Delta t)}(x)$ can be established, as follows:

for $i=0 \quad: \quad \Omega^{i}=\Omega^{(t)}, \quad Y^{i}(x)=Y^{(t)}(x)$,

for $i \rightarrow+\infty \quad: \quad \Omega^{i} \rightarrow \Omega^{(t+\Delta t)}, \quad Y^{i}(x) \rightarrow Y^{(t+\Delta t)}(x)$,

where $x \in \Omega^{(t)}$. Domains $\Omega^{(t)}$ and $Y^{(t)}(x)$ are considered as reference configurations which are not changed in the course of the iterations $i=0,1, \ldots$. To simplify the notation, in what follows, by $\boldsymbol{u}^{0, i}$ and $\boldsymbol{u}^{1, i}$ we denote displacement with respect to the fixed reference configurations at time $t$. The previous discussion yields the following two-scale computational algorithm which provides the response at time $t+\Delta t$.

Algorithm Alg-G. (Global Two-scale Algorithm).

1. Initiate. Given the reference (initial) configurations $\Omega^{(t)}$ and local configurations $\left\{Y^{(t)}(x)\right\}_{x \in \Omega^{(t)}}$, set:

(a) $i=0$. Define the new loading volume forces $\boldsymbol{f}(x):=\boldsymbol{f}^{(t+\Delta t)}(x)$ for $x \in \Omega^{(t)}$, and the boundary tractions $\boldsymbol{b}(x):=\boldsymbol{b}^{(t+\Delta t)}(x)$ for $x \in \partial_{\sigma} \boldsymbol{\Omega}^{(t)}$.

(b) For $x \in \Omega^{(t)}$, assuming $\Gamma_{*}^{(t)}$ is known in $Y_{s}^{(t)}$, define the initial trial effective stiffness $\mathbb{D}^{H, i}$, and the local mean stress $\boldsymbol{\sigma}^{i}$,

$\mathbb{D}^{H, i}(x)=\mathbb{D}^{H,(t)}(x), \quad \boldsymbol{\sigma}^{i}(x)=\boldsymbol{\sigma}^{(t)}(x), \quad \boldsymbol{u}^{0, i}=0$.

Note that $\boldsymbol{u}^{0, i}$ is the displacement with respect to configuration $\Omega^{(t)}$, so it is not the total displacement with respect to $\Omega^{(0)}$. If $t=0$, then use (4.15) and (4.16) with $\Gamma_{*}^{(0)}=\varnothing$, so that no constraints apply; obviously $\boldsymbol{\sigma}^{(0)}=0$.

2. Set $i:=i+1$. Compute the out-of-balance (residuum),

$$
r^{i}\left(\boldsymbol{v}^{0}\right):=\int_{\Omega^{(t)}} \boldsymbol{f} \cdot \boldsymbol{v}^{0}+\int_{\partial_{\sigma} \Omega^{(t)}} \boldsymbol{b} \cdot \boldsymbol{v}^{0}-\int_{\Omega^{(t)}} \boldsymbol{\sigma}^{i-1}: \boldsymbol{e}_{x}\left(\boldsymbol{v}^{0}\right) .
$$

Note that $\boldsymbol{\sigma}^{i-1}$ is the total stress with respect to the initial configuration at $t=0$.

3. Global problem: Given new “out-of-balance" $r^{i}$, find $\delta \boldsymbol{u}^{0, i} \in U_{0}\left(\Omega^{i}\right)$ which solves

$$
\int_{\Omega^{(t)}} \mathbb{D}^{H, i-1} \boldsymbol{e}_{x}\left(\delta \boldsymbol{u}^{0, i}\right): \boldsymbol{e}_{x}\left(\boldsymbol{v}^{0}\right)=r^{i}\left(\boldsymbol{v}^{0}\right) \quad \forall \boldsymbol{v}^{0} \in U_{0}\left(\Omega^{(t)}\right) .
$$

4. Update the macro-displacements, $\boldsymbol{u}^{0, i}=\boldsymbol{u}^{0, i-1}+\delta \boldsymbol{u}^{0, i}$.

5. Local problems: for a.a. $x \in \Omega^{(i)}$, find $\boldsymbol{u}^{1, i}(x, \cdot) \in \mathcal{K}_{Y}\left(x, \nabla_{\chi} \boldsymbol{u}^{0, i}\right)$ satisfying

$f_{Y_{s}^{(t)}(x)} \mathbb{D} \boldsymbol{e}_{y}\left(\boldsymbol{u}^{1, i}+\Pi^{i j} e_{i j}^{x}\left(\boldsymbol{u}^{0, i}\right)\right): \boldsymbol{e}_{y}\left(\boldsymbol{v}-\boldsymbol{u}^{1, i}\right) \geq 0, \quad \forall \boldsymbol{v} \in \mathcal{K}_{Y}\left(x, \nabla_{x} \boldsymbol{u}^{0, i}\right)$, where $\mathcal{K}_{Y}\left(x, \nabla_{x} \boldsymbol{w}\right)=\left\{\boldsymbol{v} \in \mathbf{H}_{\#}^{1}\left(Y_{s}^{(t)}\right) \mid \quad g_{c}^{(t)}(\boldsymbol{v}, \nabla \boldsymbol{w}) \leq 0\right\}$ with $g_{c}^{(t)}(\boldsymbol{v}, \nabla \boldsymbol{w})=\left[\boldsymbol{v}+\nabla_{x} \boldsymbol{w} y-y\right]_{n}^{Y}$.

The solution yields the true contact boundary $\Gamma_{*}^{i,(t)}$ by virtue of the active constraint set, see below.

6. Update micro-configurations and the total stress for all $x \in \Omega^{(t)}$, 
$\boldsymbol{u}^{\mathrm{mic}, i}(x, y)=\boldsymbol{u}^{1, i}(x, y)+\nabla_{x} \boldsymbol{u}^{0, i}(x) \widehat{y}, \quad y \in Y_{s}^{(t)}(x)$,

$\boldsymbol{\sigma}^{i,(t)}(x)=\boldsymbol{\sigma}^{(t)}(x)+f_{Y_{s}^{(t)}(x)} \mathbb{D e}_{y}\left(\boldsymbol{u}^{\mathrm{mic}, i}\right)(x, \cdot)$,

$\Gamma_{*}^{i,(t)}(x)=\left\{x^{ \pm} \in \Gamma_{ \pm}^{(t)} \mid\left[\nabla_{x} \boldsymbol{u}^{0, i} \widehat{y}+\boldsymbol{u}^{1, i}-\widehat{y}\right]_{n}^{i,(t)}=0\right\}$.

7. Compute the consistent tangent modulus $\mathbb{D}^{H, i+1}$ for all $x \in \Omega^{(t)}$. By virtue of (4.4) and $\Gamma_{*}^{i,(t)}(x)$, define

$V_{0}\left(\boldsymbol{U}, Y_{s}^{i,(t)}, x\right)=\left\{\boldsymbol{v} \in \mathbf{H}_{\#}^{1}\left(Y_{s}^{(t)}\right) \mid\left[\boldsymbol{v}+\boldsymbol{U}_{n}^{i,(t)}=0\right.\right.$ on $\left.\Gamma_{*}^{i,(t)}(x)\right\}$.

Corrector problem: Find $\boldsymbol{w}^{k l} \in V_{0}\left(\boldsymbol{\Pi}^{k l}, Y_{s}^{i,(t)}, x\right)$, such that

$f_{Y_{s}^{(t)}(x)} \mathbb{D} \boldsymbol{e}_{y}\left(\boldsymbol{w}^{k l}+\Pi^{k l}\right): \boldsymbol{e}_{y}(\boldsymbol{v})=0, \forall \boldsymbol{v} \in V_{0}\left(0, Y_{s}^{i,(t)}, x\right)$.

Compute the effective tangent modulus,

$D_{r s k l}^{H, i}(\boldsymbol{x})=f_{Y_{s}^{(t)}(x)} \mathbb{D} \boldsymbol{e}_{y}\left(\boldsymbol{w}^{k l}+\boldsymbol{\Pi}^{k l}\right): \boldsymbol{e}_{y}\left(\boldsymbol{w}^{r s}+\boldsymbol{\Pi}^{r s}\right)$.

8. Stop condition: if the dual norm of $r^{i}$ and the increments $\delta \boldsymbol{u}^{i}$ are small enough,

$\max _{\boldsymbol{v} \in U_{0}\left(\Omega^{(t)}\right)} r^{i}(\boldsymbol{v}) /\|\boldsymbol{v}\|_{\mathbf{H}^{1}\left(\Omega^{(t)}\right)} \leqslant \epsilon_{1}, \quad\left\|\delta \boldsymbol{u}^{0, i}\right\|_{\mathbf{H}^{1}\left(\Omega^{(t)}\right)} \leqslant \epsilon_{2}$

then STOP, otherwise GO TO Step 2.

\section{Remark 1.}

(i) Note that $\left[\boldsymbol{u}^{1, i}+\left(\nabla_{x} \boldsymbol{u}^{0, i}-\boldsymbol{I}\right) \widehat{y}\right]_{n}^{i,(t)}=0$ due to the true contact on $\Gamma_{*}^{i}(x)$ at iteration $i$, hence it holds that (see also (4.4)),

$$
\begin{aligned}
g_{*}^{Y(t)}\left(\boldsymbol{v}+\boldsymbol{U}+\boldsymbol{u}^{1, i}, \nabla_{x} \boldsymbol{u}^{0, i}\right) & =\left[\boldsymbol{v}+\boldsymbol{U}+\boldsymbol{u}^{1, i}+\left(\nabla_{x} \boldsymbol{u}^{0, i}-\boldsymbol{I}\right) \widehat{y}\right]_{n}^{i,(t)} \\
& =[\boldsymbol{v}+\boldsymbol{U}]_{n}^{i,(t)}
\end{aligned}
$$

The true contact boundary $\Gamma_{*}^{i}$ in the deformed configuration can be determined,

$$
\begin{aligned}
& \Gamma_{ \pm}^{i,(t)}=\Gamma_{ \pm}^{(t)}+\left\{\boldsymbol{u}^{\mathrm{mic}, i}(x, y)\right\}, \\
& \Gamma_{*}^{i}=\Gamma_{+}^{i,(t)} \cap \Gamma_{-}^{i,(t)} .
\end{aligned}
$$

(ii) To solve the nonlinear problem (4.13) for $\boldsymbol{u}^{1, i}$, one can start with the initial guess based on the preceding iteration $\boldsymbol{u}^{1, i-1}$. Besides the Stop conditions in (4.17) one should check also the convergences, $\left\|\boldsymbol{u}^{1, i}-\boldsymbol{u}^{1, i-1}\right\|_{\mathbf{H}_{* t}^{1}\left(Y_{s}^{(t)}\right)} \rightarrow 0$.

(iii) The "sticking" constraint imposed on $\Gamma_{*}^{i,(t)}(x)$ by virtue of the admissibility set $V_{0}\left(\boldsymbol{U}, Y_{s}^{i,(t)}, x\right)$ can be enforced by the Lagrangian penalty, or by the master-slave conception.

(iv) As an alternative of the loading by volume forces and boundary tractions, the algorithm can be modified easily for the structure loading by non-homogeneous Dirichlet conditions, prescribing nonvanishing displacements on a subpart of $\partial_{u} \Omega$.

In the following text, we drop the superscript ${ }^{(t)}$ labeling the time level $t$. The reference configurations $Y_{s}^{(t)}$ and $\Omega^{(t)}$ will be abbreviated by $Y_{S}$ and $\Omega$, respectively, so the presented formulations are associated with computing "one time increment" in the context of the Algorithm presented above.

\section{Microstructures with rigid inclusions}

For the rest of the paper we shall confine to microstructures with rigid inclusions such that the unilateral contact can appear on a part of the inclusions surfaces, see Fig. 3. In this setting, the solid part $Y_{S}$ in reference cell $Y$ consists of the compliant elastic part $Y_{S} \subset Y_{S}$ and of a rigid inclusion $Y_{R} \subset Y_{S}$, whereby $Y_{R}=Y_{S} \backslash \overline{Y_{S}}$. For the sake of clarity, below we employ $Y_{S R} \equiv Y_{S}$ to refer to the whole solid part of $Y$. We assume that $\Gamma_{C R}$ is a contact boundary segment on the rigid inclusion, and denote by $\Gamma_{c s}$ the other contact surface, so that the self-contact in the solid part $Y_{s}$ is attained on matching subparts $\Gamma_{c R}^{*}$ and $\Gamma_{c S}^{*}$ of the rigid and elastic phases, respectively. In the context of the notation (2.4), $\Gamma_{c S}=\Gamma_{-}^{Y}$ and $\Gamma_{c R}=\Gamma_{+}^{Y}$. Below, by $\Gamma_{c}$ we refer to both surfaces $\Gamma_{c S}$ and $\Gamma_{c R}$. by $\Gamma_{*}$ we denote the actual true contact surface defined in the sense of (4.4). Furthermore, by $\Gamma_{S R}=\partial Y_{S} \cap \partial Y_{R}$ we denote the interface between the elastic and rigid parts.

Let us define the space of rigid body motions (RBM), $\boldsymbol{W}_{\mathrm{RBM}}\left(Y_{R}\right)=\left\{\boldsymbol{v} \in \mathbf{H}^{1}\left(Y_{R}\right) \mid \boldsymbol{v}=\boldsymbol{R} \overline{\boldsymbol{\varphi}}+\overline{\boldsymbol{v}}, \overline{\boldsymbol{\varphi}}, \overline{\boldsymbol{v}} \in \mathbb{R}^{3}\right\}$, where the rotation matrix $\boldsymbol{R}$ is established with respect to a reference point which can be associated with cell barycenter $\hat{y}$, so that

$\boldsymbol{R}=\left[\begin{array}{lll}0 & \widehat{y}_{3} & -\widehat{y}_{2} \\ -\widehat{y}_{3} & 0 & \widehat{y}_{1} \\ \hat{y}_{2} & -\widehat{y}_{1} & 0\end{array}\right], \quad y \in Y_{R}$.

Thus, any $\boldsymbol{w} \in \boldsymbol{W}_{\mathrm{RBM}}\left(Y_{R}\right)$ is equivalently represented by a couple $(\overline{\boldsymbol{\varphi}}, \overline{\boldsymbol{v}}) \in \mathbb{R}^{3} \times \mathbb{R}^{3}$, where $\overline{\boldsymbol{\varphi}}$ is the rotation angle and $\overline{\boldsymbol{v}}$ is the translation.

Further we introduce the extension operator $\mathcal{P}_{Y_{R}}$ which associates any RBM defined in $Y_{R}$ with its smooth extension to $\mathbf{H}^{1}\left(Y_{S}\right)$, thus, for any $\overline{\boldsymbol{\varphi}}, \overline{\boldsymbol{v}} \in \mathbb{R}^{3}$,

$\mathcal{P}_{Y_{R}}:(\overline{\boldsymbol{\varphi}}, \overline{\boldsymbol{v}}) \mapsto\left\{\boldsymbol{v} \in \mathbf{H}^{1}\left(Y_{S R}\right), \quad \boldsymbol{v}=\boldsymbol{R} \overline{\boldsymbol{\varphi}}+\overline{\boldsymbol{v}}\right.$ in $\left.Y_{R}\right\}$.

As a counterpart to the extension operation due to (5.2), by $\boldsymbol{\mathcal { R }}_{Y_{R}}$ we denote the restriction to functions described in $Y_{R}$ by the RBM modes only. This allows us to define the following restriction space

$\mathcal{R}_{Y_{R}} \mathbf{H}^{1}(Y)=\left\{\boldsymbol{v} \in \mathbf{H}^{1}(Y) \mid \exists \boldsymbol{w} \in \boldsymbol{W}_{\mathrm{RBM}}\left(Y_{R}\right): \boldsymbol{v}=\mathcal{P}_{Y_{R}}(\boldsymbol{w})\right\}$.

Thus, for any $\boldsymbol{v} \in \mathcal{R}_{Y_{R}} \mathbf{H}^{1}(Y)$ there is a couple $\boldsymbol{w}=(\overline{\boldsymbol{\psi}}, \overline{\boldsymbol{v}})$ such that $\boldsymbol{v}=\mathcal{P}_{Y_{R}}(\boldsymbol{w})$; we write $\boldsymbol{w}=\mathcal{P}_{Y_{R}}^{-1}(\boldsymbol{v})$.

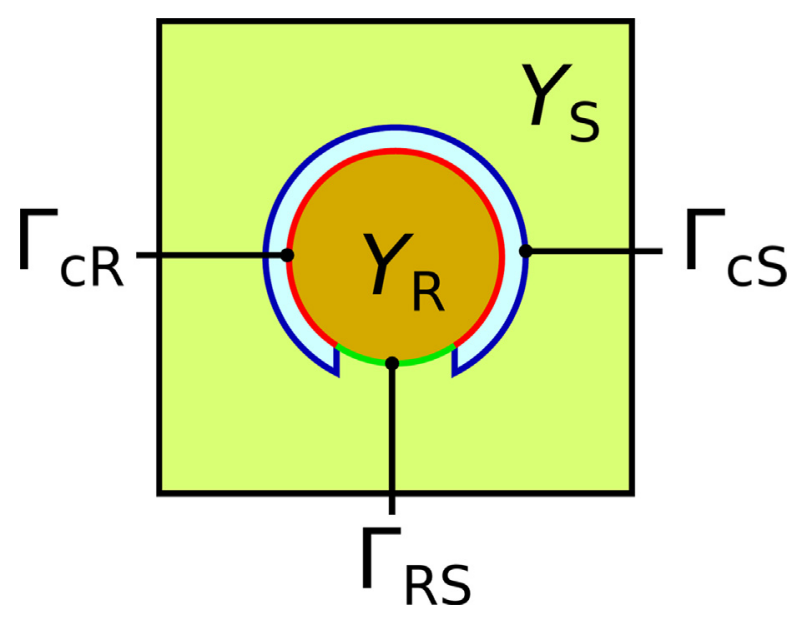

Fig. 3. Scheme of the representative cell $Y$ with labelled contact surfaces $\Gamma_{c S}, \Gamma_{c R}$ and the interface $\Gamma_{R S}$ on which the rigid inclusion $Y_{R}$ is clamped into the compliant part $Y_{S}$. The gap between $\Gamma_{c S}$ and $\Gamma_{c R}$ is very small in the geometry employed in the numerical examples, see Fig. 4. 


\subsection{Unilateral contact problem}

To establish this problem arising from (4.13) for the contact with rigid inclusions, we need to define the gap function and to introduce the set of admissible displacements. First we establish the homologous points on $\Gamma_{c R}$ and $\Gamma_{c S}$.

Definition 3. Two points $y^{R} \in \Gamma_{c R}$ and $y^{S} \in \Gamma_{c S}$ are the homologous contact points, iff $\exists t \geqslant 0$ such that $y^{S}=y^{R}-\operatorname{tn}^{c}\left(y^{R}\right)$, where $\boldsymbol{n}^{c}\left(y^{R}\right)$ is the unit vector normal at $y^{R}$ to surface $\partial Y_{R}$ and pointing inward to $Y_{R}$. To refer to $y^{R}$ as the homologous point to $y^{S}$, we write $y^{R}=\mathcal{H}\left(y^{S}\right)$. By $y \in \Gamma_{c}$ we mean the two homologous points $y^{R}$ and $y^{S}$.

We assume that for any $y^{S} \in \Gamma_{c S}$, there is just one $y^{R}=\mathcal{H}\left(y^{S}\right)$. The contact gap at the reference configuration is $s^{c}\left(y^{S}\right)=\left|y^{R}-y^{S}\right|=\left|\widehat{y}^{R}-\widehat{y}^{S}\right|$.

For a given macroscopic strain tensor $\boldsymbol{G}=\boldsymbol{G}^{T}, \boldsymbol{G}=\left(G_{i j}\right)$, the gap function is introduced, as follows,

$g_{c}^{R S}(\boldsymbol{u}, \boldsymbol{G}, \overline{\boldsymbol{\varphi}}, \overline{\boldsymbol{v}}):=\boldsymbol{n}^{c} \cdot\left(\boldsymbol{u}\left(y^{S}\right)+\boldsymbol{G} \widehat{y}^{S}-\boldsymbol{R}\left(\mathcal{H}\left(y^{S}\right)\right) \overline{\boldsymbol{\varphi}}-\overline{\boldsymbol{v}}\right)-s^{c}\left(y^{S}\right)$,

$y^{S} \in \Gamma_{c S}$.

Then, we define

$$
\begin{aligned}
\mathcal{K}^{R S}(\boldsymbol{G})= & \left\{\boldsymbol{v} \in \mathbf{H}_{\#}^{1}\left(Y_{S}\right) \mid \boldsymbol{v}+\boldsymbol{U} \in \mathcal{R}_{Y_{R}} \mathbf{H}^{1}\left(Y_{S R}\right),\right. \text { and } \\
& g_{c}^{R S}(\boldsymbol{v}, \boldsymbol{G}, \overline{\boldsymbol{\varphi}}, \overline{\boldsymbol{v}}) \leqslant 0 \text { on } \Gamma_{c}, \\
& \text { where } \left.\boldsymbol{U}=\boldsymbol{G} \hat{y} \quad \text { in } Y_{S}, \text { and }(\overline{\boldsymbol{\varphi}}, \overline{\boldsymbol{v}})=\mathcal{P}_{Y_{R}}^{-1}(\boldsymbol{v}+\boldsymbol{U})\right\} .
\end{aligned}
$$

Note that any $\boldsymbol{u} \in \mathcal{K}^{R S}(\boldsymbol{G})$ is $Y$-periodic and represents also the rigid displacement in $Y_{R}$, such that $(\overline{\boldsymbol{\varphi}}, \overline{\boldsymbol{v}})=\mathcal{P}_{Y_{R}}^{-1}(\boldsymbol{u}+\boldsymbol{G} \hat{y})$. By virtue of (5.2), the jump of traces vanishes on the two faces of the interface $\Gamma_{R S}$, thus $0=\left[\boldsymbol{\mathcal { R }}_{Y_{R}} \boldsymbol{v}\right]^{\Gamma_{S R}}=\boldsymbol{u}(y)+\boldsymbol{G} \hat{y}-\boldsymbol{R}(\mathcal{H}(y)) \overline{\boldsymbol{\varphi}}-\overline{\boldsymbol{v}}$ for $y \in \Gamma_{R S}$ and $\boldsymbol{v} \in \mathcal{K}^{R S}(\boldsymbol{G})$.

Problem (4.13) reads, as follows: For a given $\boldsymbol{G}^{0}:=\left(e_{i j}^{x}\left(\boldsymbol{u}^{0}\right)\right)$, find $\boldsymbol{u}^{1} \in \mathcal{K}^{R S}\left(\boldsymbol{G}^{0}\right)$, such that

$f_{Y_{S}} \mathbb{D} \boldsymbol{e}_{y}\left(\boldsymbol{u}^{1}+\boldsymbol{G}^{0} \hat{y}\right): \boldsymbol{e}_{y}\left(\boldsymbol{v}-\boldsymbol{u}^{1}\right) \geq 0, \quad \forall \boldsymbol{v} \in \mathcal{K}^{R S}\left(\boldsymbol{G}^{0}\right)$.

The integration (5.6) is only in the compliant part. Note that the RBM displacements in $Y_{R}$ are obtained due to $\boldsymbol{u}^{1}$ and the definition of $\mathcal{K}^{R S}(\boldsymbol{G})$. In the discretized formulation, we shall treat the RBM part of the displacement explicitly.

\subsection{Tangent stiffness and the bilateral contact problem}

To compute the tangent stiffness according to (4.15) and (4.16), we now state the local linear problem for corrector functions with the bilateral contact on the current true contact interface $\Gamma_{*}$. For brevity, in what follows, the iteration index $i$ involved in $A l g-G$ is omitted. The set $V_{0}$ employed in (4.15) must be adapted to capture the RBM in the inclusions, so that we define

$g_{*}^{R S}(\boldsymbol{u}, \boldsymbol{G}, \overline{\boldsymbol{\varphi}}, \overline{\boldsymbol{v}}):=\boldsymbol{n}^{*} \cdot\left(\boldsymbol{u}\left(y^{S}\right)+\boldsymbol{G} \widehat{y}^{S}-\boldsymbol{R}\left(\mathcal{H}\left(y^{S}\right)\right) \overline{\boldsymbol{\varphi}}-\overline{\boldsymbol{v}}\right), \quad y^{S} \in \Gamma_{c S}$,

where $\boldsymbol{n}^{*}$ is normal to $\Gamma_{*}$, and introduce

$$
\begin{aligned}
\mathcal{V}^{R S}(\boldsymbol{G})= & \left\{\boldsymbol{v} \in \mathbf{H}_{\#}^{1}\left(Y_{S}\right) \mid \boldsymbol{v}+\boldsymbol{U} \in \mathcal{R}_{Y_{R}} \mathbf{H}^{1}\left(Y_{S R}\right),\right. \\
& \text { and } g_{*}^{R S}(\boldsymbol{v}, \boldsymbol{G}, \overline{\boldsymbol{\varphi}}, \overline{\boldsymbol{v}})=0 \text { on } \Gamma_{c}, \\
& \text { where } \left.\boldsymbol{U}=\boldsymbol{G} \hat{y} \quad \text { in } Y_{S}, \text { and }(\overline{\boldsymbol{\varphi}}, \overline{\boldsymbol{v}})=\mathcal{P}_{Y_{R}}^{-1}(\boldsymbol{v}+\boldsymbol{U})\right\} .
\end{aligned}
$$

The corrector functions satisfy the following problem: Find $\boldsymbol{\omega}^{i j} \in \mathcal{V}^{R S}\left(\boldsymbol{G}^{i j}\right)$ with $\boldsymbol{G}^{i j}=\left(G_{k l}^{i j}\right), G_{k l}^{i j}=\delta_{i k} \delta_{j l}$, such that

$f_{Y_{S}} \mathbb{D} \boldsymbol{e}_{y}\left(\boldsymbol{\omega}^{i j}+\Pi^{i j}\right): \boldsymbol{e}_{y}(\boldsymbol{v})=0, \quad \forall \boldsymbol{v} \in \mathcal{V}^{R S}(\boldsymbol{0})$.

Problem (5.9) involves also the rigid body displacements defined in $Y_{R}$ by $\left(\overline{\boldsymbol{w}}^{i j}, \overline{\boldsymbol{\varphi}}^{i j}\right)=\mathcal{P}_{Y_{R}}^{-1}\left(\boldsymbol{\omega}^{i j}+\boldsymbol{\Pi}^{i j}\right)$, whereby the interface conditions hold,

$$
\begin{array}{ll}
\boldsymbol{\omega}^{i j}+\Pi^{i j}-\boldsymbol{R} \overline{\boldsymbol{\varphi}}^{i j}-\overline{\boldsymbol{w}}^{i j}=0 & \text { on } \Gamma_{R S}, \\
\boldsymbol{n}^{*} \cdot\left(\boldsymbol{\omega}^{i j}+\boldsymbol{\Pi}^{i j}-\boldsymbol{R} \overline{\boldsymbol{\varphi}}^{i j}-\overline{\boldsymbol{w}}^{i j}\right)=0 & \text { on } \Gamma_{*} .
\end{array}
$$

In analogy, the test functions $\boldsymbol{v}$ used in (5.9) satisfy $\boldsymbol{n}^{*} \cdot\left(\left.\boldsymbol{v}\right|_{\Gamma_{c S}^{*}}-\boldsymbol{R} \overline{\boldsymbol{\psi}}-\overline{\boldsymbol{v}}\right)=0$ on $\Gamma_{*}$ and $\left.\boldsymbol{v}\right|_{\Gamma_{c S}^{*}}-\boldsymbol{R} \overline{\boldsymbol{\psi}}-\overline{\boldsymbol{v}}=\mathbf{0}$ on $\Gamma_{R S}$, where $(\overline{\boldsymbol{\psi}}, \overline{\boldsymbol{v}})=\mathcal{P}_{Y_{R}}^{-1}(\boldsymbol{v})$. Obviously, the tangent modulus is computed by (4.16), since only the integration over the compliant part $Y_{S}$ applies.

\section{FEM discretized problem}

In this section we deal with the discretized problems arising from the local problems presented in the previous section. The main purpose here is to propose a numerical method to solve the local unilateral self-contact problem (5.6) without any regularization. The algorithm is based on the semi-smooth Newton method which is well suited for solving the nonlinear implicit complementarity problems, [8].

We use the standard matrix notation at the global level of the FE model. In this context, by $\overline{\mathbf{A}}$ we denote a (square) matrix with all degrees of freedom (DOFs), so that the rows and columns refer to all nodes with all DOFs regardless boundary conditions (BCs). By $(\overline{\mathbf{A}})^{\#}$ we designate the matrix derived from $\overline{\mathbf{A}}$ by reducing all "slave" DOFs due to the periodic BCs. We consider $\mathbf{A}=(\overline{\mathbf{A}})^{\#}$. Analogous notation is adopted for general non-square matrices, or vectors, if the row, or column positions are related to DOFs influenced by prescribing periodic, or the Dirichlet type BCs. Also the DOFs associated with the contact boundary can be expressed in terms of the local coordinate systems aligned with the normal and tangential directions of the boundary; matrices and vectors constituted by such rotated coordinates will be denoted by tilde, i.e. $\tilde{\mathbf{A}}$. Obviously, in what follows, by "vector" we mean the onecolumn matrix, whereby $\mathbf{v}^{T}$ is the corresponding row-vector obtained by transposing $\mathbf{v}$.

\subsection{Discretized problem formulation for computing the tangent stiffness}

Without any additional constraints, thus ignoring all constraints arising from restriction of the solutions by the set $\mathcal{V}^{R S}(\boldsymbol{G})$ defined in (5.8), discretized problem (5.9) writes

$$
\mathbf{v}^{T}\left[\mathbf{K} \mathbf{u}^{i j}+\left(\overline{\mathbf{K}} \overline{\mathbf{z}}^{i j}\right)^{\#}\right]=0, \quad \text { where }\left.\overline{\mathbf{z}}^{i j}\right|_{\text {node }}=\left[\begin{array}{l}
y_{j} \delta_{1 i} \\
y_{j} \delta_{2 i} \\
y_{j} \delta_{3 i}
\end{array}\right],
$$

and the column $\mathbf{v}$ is the vector associated with a test function nonrestricted by $\mathcal{V}^{R S}(\boldsymbol{0})$; By $\left.\right|_{\text {node }}$ we refer to DOFs at a FE node. The constrained imposed by virtue of the set $\mathcal{V}^{R S}$ will now be applied explicitly.

To express the RBM modes, we introduce matrix $\mathbf{B}=[\mathbf{R}, \mathbf{Y}]$, where $\mathbf{R}$ is formed as a column of $3 \times 3$ blocks of $\boldsymbol{R}$ defined in (5.1), and $\mathbf{Y}$ is formed as a column of $3 \times 3$ blocks of the identity 
I. Let $\mathbf{u}_{R}$ be a subvector of unknowns associated with the DOFs on $\Gamma_{R S}$. By virtue of the RBM, column vector $\mathbf{p}=[\overline{\boldsymbol{\varphi}} ; \overline{\boldsymbol{w}}]$ is introduced which involves variables of the rotation and translation DOFs describing the RBM of the rigid part, $Y_{R}$. In particular, Bp yields displacement DOFs on the rigid inclusion boundary, so that discretized $(5.10)_{1}$ yields

$\mathbf{u}_{R}^{i j}+\mathbf{z}_{R}^{i j}=\mathbf{B}_{R} \mathbf{p}^{i j}$,

where ${ }^{i j}$ refers to the macroscopic strain mode, and $\mathbf{B}_{R}$ is constituted by DOFs on $\Gamma_{R S}$. To simplify notation, we employ

$\mathbf{g}^{i j}=\left(\overline{\mathbf{K}}^{i j}\right)^{\#}$,

which represents the discretized stresses induced by the macroscopic strain mode, $\mathbb{D} \boldsymbol{e}_{y}\left(\boldsymbol{\Pi}^{k l}\right)$.

Furthermore we shall assume that all DOFs are expressed with respect to local coordinate systems introduced on the contact boundary. By $\mathbf{u}_{t}$ and $\mathbf{u}_{n}$ we refer to the "tangential" and "normal" DOFs, respectively, at the nodes on the contact boundary $\Gamma_{c s}$; recall that the normal direction are determined with respect to the contact surface $\Gamma_{c R}$ on the rigid part $Y_{R}$, in accordance with the definition of $\boldsymbol{n}^{c}$, see Definition 3. Specifically, since the bilateral contact is relevant to the actual "true" contact boundary $\Gamma_{*}$, only those normal DOFs at the mesh nodes of $\Gamma_{c S}^{*} \subset \Gamma_{c S}$ are involved in the bilateral contact condition. However, the decomposition into the tangential and normal displacements is introduced along entire $\Gamma_{c}$ by virtue of the normal vector $\boldsymbol{n}^{c}$ employed in Definition 3, when dealing with the unilateral contact. By $\mathbf{Q}$ we denote the global rotation matrix which is block-diagonal; each 3-by-3 block is either identity, or the rotation ortho-normal matrix defined by normal vector and two tangent directions associated with surfaces of $\Gamma_{c}$. The matrix object expressed with respect to the rotated coordinate systems will be denoted by $\widetilde{\text {, so that }} \widetilde{\mathbf{u}}=\mathbf{Q \mathbf { u }}$ contains all DOFs defined with respect to the local rotated coordinate systems.

In this context, we shall employ the following notation:

$$
\widetilde{\mathbf{K}}=\mathbf{Q K} \mathbf{Q}^{T}, \widetilde{\mathbf{u}}=\mathbf{Q u}, \quad \widetilde{\mathbf{B}}=\mathbf{Q B}, \quad \widetilde{\mathbf{u}}_{n}=\mathbf{X}_{n}^{*} \widetilde{\boldsymbol{u}}, \quad \widetilde{\mathbf{u}}_{t}=\mathbf{X}_{t}^{*} \widetilde{\boldsymbol{u}}
$$

where matrices $\mathbf{X}_{n}^{*}$ and $\mathbf{X}_{t}^{*}$ select the normal and the tangential DOFs, respectively, for the actual true contact boundary nodes on $\Gamma_{c S}^{*}$.

We assume that the rotation operation, i.e. $\mathbf{Q K}$, or $\mathbf{Q B}$, is commutative with the restriction/extension by the DOFs on boundary $\partial Y$ due to the periodic BCs. Further, we define the following notation:

- $\widetilde{\mathbf{u}}_{n}$ involves the co-normal DOFs of $\mathbf{u}$ on the true contact boundary $\Gamma_{c S}^{*}$.

- $\widetilde{\mathbf{u}}_{R}=\widetilde{\mathbf{B}}_{R} \mathbf{p}-\widetilde{\mathbf{z}}_{R}$ contains the RBM DOFs on $\Gamma_{R S}$.

- $\widetilde{\mathbf{u}}_{A}$ involves all DOFs of $\widetilde{\mathbf{u}}$, but the normal DOFs $\widetilde{\mathbf{u}}_{n}$ and the RBM DOFs on $\Gamma_{R S}$.

- $\widetilde{\mathbf{B}}_{n}=\mathbf{X}_{n} \widetilde{\mathbf{B}}_{c}=\mathbf{X}_{n} \mathbf{Q} \mathbf{B}_{c}$ contains the RBM DOFs aligned with the normal direction (at each node) on $\Gamma_{c s}$. Matrix $\mathbf{B}_{c}$ is composed of DOFs on $\Gamma_{c s}$. Obviously, $\widetilde{\mathbf{B}}_{n}^{*}$ is related to the true contact boundary $\Gamma_{c S}^{*}$, while $\widetilde{\mathbf{B}}_{n}$ is related to entire $\Gamma_{c s}$; the latter option is employed when dealing with the unilateral contact problem.

Using the notation above introduced, the system (6.1) can be rewritten, as follows,

$$
\left[\tilde{\mathbf{v}}_{A}^{T}, \tilde{\mathbf{v}}_{R}^{T}, \tilde{\mathbf{v}}_{n}^{T}\right] \cdot\left(\left[\begin{array}{ccc}
\tilde{\mathbf{K}}_{A A} & \tilde{\mathbf{K}}_{A R} & \tilde{\mathbf{K}}_{A n} \\
\tilde{\mathbf{K}}_{R A} & \tilde{\mathbf{K}}_{R R} & \tilde{\mathbf{K}}_{R n} \\
\tilde{\mathbf{K}}_{n A} & \tilde{\mathbf{K}}_{n R} & \tilde{\mathbf{K}}_{n n}
\end{array}\right]\left[\begin{array}{c}
\tilde{\mathbf{u}}_{A} \\
\tilde{\mathbf{u}}_{R} \\
\tilde{\mathbf{u}}_{n}
\end{array}\right]+\left[\begin{array}{c}
\tilde{\mathbf{g}}_{A} \\
\tilde{\mathbf{g}}_{R} \\
\tilde{\mathbf{g}}_{n}
\end{array}\right]\right)=0,
$$

where the superscripts ${ }^{i j}$ associated with the strain modes were dropped. Both the test functions $\tilde{\mathbf{v}}_{R}^{T}, \tilde{\mathbf{v}}_{n}^{T}$ and the unknowns $\tilde{\mathbf{u}}_{R}^{T}, \tilde{\mathbf{u}}_{n}^{T}$ are subject to the RBM constraints deduced from (5.10), namely (6.2),

$$
\begin{aligned}
& \tilde{\mathbf{u}}_{R}^{i j}+\tilde{\mathbf{z}}_{R}^{i j}=\tilde{\mathbf{B}}_{R} \mathbf{p}^{i j}, \\
& \tilde{\mathbf{v}}_{R}=\tilde{\mathbf{B}}_{R} \mathbf{q}, \\
& \tilde{\mathbf{u}}_{n}^{i j}+\tilde{\mathbf{z}}_{n}^{i j}=\tilde{\mathbf{B}}_{n} \mathbf{p}^{i j}, \\
& \tilde{\mathbf{v}}_{n}=\tilde{\mathbf{B}}_{n} \mathbf{q},
\end{aligned}
$$

Hence, upon substituting (6.6) in (6.5), we get

$$
\begin{aligned}
\tilde{\mathbf{v}}_{A}^{T} & \cdot\left(\tilde{\mathbf{K}}_{A A} \tilde{\mathbf{u}}_{A}+\tilde{\mathbf{K}}_{A R}\left(\tilde{\mathbf{B}}_{R} \mathbf{p}-\tilde{\mathbf{z}}_{R}\right)+\tilde{\mathbf{K}}_{A n}\left(\tilde{\mathbf{B}}_{n} \mathbf{p}-\tilde{\mathbf{z}}_{n}\right)+\tilde{\mathbf{g}}_{A}\right) \\
& +\mathbf{q}^{T} \tilde{\mathbf{B}}_{R}^{T} \cdot\left(\tilde{\mathbf{K}}_{R A} \tilde{\mathbf{u}}_{A}+\tilde{\mathbf{K}}_{R R}\left(\tilde{\mathbf{B}}_{R} \mathbf{p}-\tilde{\mathbf{z}}_{R}\right)+\tilde{\mathbf{K}}_{R n}\left(\tilde{\mathbf{B}}_{n} \mathbf{p}-\tilde{\mathbf{z}}_{n}\right)+\tilde{\mathbf{g}}_{R}\right) \\
& +\mathbf{q}^{T} \tilde{\mathbf{B}}_{n}^{T} \cdot\left(\tilde{\mathbf{K}}_{n A} \tilde{\mathbf{u}}_{A}+\tilde{\mathbf{K}}_{n R}\left(\tilde{\mathbf{B}}_{R} \mathbf{p}-\tilde{\mathbf{z}}_{R}\right)+\tilde{\mathbf{K}}_{n n}\left(\tilde{\mathbf{B}}_{n} \mathbf{p}-\tilde{\mathbf{z}}_{n}\right)+\tilde{\mathbf{g}}_{n}\right)=0,
\end{aligned}
$$

which must hold for arbitrary test vectors $\tilde{\mathbf{v}}$. Thereby, (6.7) can be rewritten in a more compact way,

$$
\begin{aligned}
& {\left[\begin{array}{ll}
\tilde{\mathbf{K}}_{A A} & \tilde{\mathbf{K}}_{A R} \tilde{\mathbf{B}}_{R}+\tilde{\mathbf{K}}_{A n} \tilde{\mathbf{B}}_{n} \\
\tilde{\mathbf{B}}_{R}^{T} \tilde{\mathbf{K}}_{R A}+\tilde{\mathbf{B}}_{n}^{T} \tilde{\mathbf{K}}_{n A} & \tilde{\mathbf{B}}_{R}^{T} \tilde{\mathbf{K}}_{R R} \tilde{\mathbf{B}}_{R}+\tilde{\mathbf{B}}_{R}^{T} \tilde{\mathbf{K}}_{R n} \tilde{\mathbf{B}}_{n}+\tilde{\mathbf{B}}_{n}^{T} \tilde{\mathbf{K}}_{n R} \tilde{\mathbf{B}}_{R}+\tilde{\mathbf{B}}_{n}^{T} \tilde{\mathbf{K}}_{n n} \tilde{\mathbf{B}}_{n}
\end{array}\right]\left[\begin{array}{l}
\tilde{\mathbf{u}}_{A} \\
\mathbf{p}
\end{array}\right]} \\
& =\left[\begin{array}{l}
\tilde{\mathbf{K}}_{A R} \tilde{\mathbf{z}}_{R}+\tilde{\mathbf{K}}_{A n} \tilde{\mathbf{z}}_{n}-\tilde{\mathbf{g}}_{A} \\
\left(\tilde{\mathbf{B}}_{R}^{T} \tilde{\mathbf{K}}_{R R}+\tilde{\mathbf{B}}_{n}^{T} \tilde{\mathbf{K}}_{n R}\right) \tilde{\mathbf{z}}_{R}+\left(\tilde{\mathbf{B}}_{R}^{T} \tilde{\mathbf{K}}_{R n}+\tilde{\mathbf{B}}_{n}^{T} \tilde{\mathbf{K}}_{n n}\right) \tilde{\mathbf{z}}_{n}-\tilde{\mathbf{B}}_{R}^{T} \tilde{\mathbf{g}}_{R}-\tilde{\mathbf{B}}_{n}^{T} \tilde{\mathbf{g}}_{n}
\end{array}\right] .
\end{aligned}
$$

To evaluate the tangential homogenized elasticity according to (4.16), we can proceed by pursuing the standard procedure, provided $\tilde{\mathbf{u}}=\left[\tilde{\mathbf{u}}_{A} ; \tilde{\mathbf{u}}_{R}, \tilde{\mathbf{u}}_{n}\right]$ is computed from (6.6) $)_{1,3}$. Then, using the notation $\overline{\mathbf{u}}$ designating the extended column vector of all DOFs, including the "slave" DOFs of the periodic BCs,

$\mathrm{D}_{i j k l}^{H}=\left[\overline{\mathbf{u}}^{k l}+\overline{\mathbf{z}}^{k l}\right]^{T} \overline{\mathbf{K}}\left[\overline{\mathbf{u}}^{i j}+\overline{\mathbf{z}}^{i j}\right]$.

Recall that, when dealing with the extended vectors and matrices, the rotation operation associated with local coordinate system is assumed to be independent of the extension.

\subsection{Discretized problem formulation for solving the unilateral contact problem}

Here we deal with the FE discretized contact problem (5.6). We employ the same decomposition of the DOFs of the compliant solid part in $Y_{S}$, as it has been applied in problem (6.8). However, now the normal and tangent displacement DOFs are considered along the entire contact surface $\Gamma_{c s}$; note that in the bilateral contact problem, the decomposition was introduced on the true contact surface $\Gamma_{c S}^{*}$ only.

For the FE-discretized formulation, some specific notation in the context of the contact problem (3.6) will be employed. By the relation $\mathbf{a} \stackrel{\text { FEM }}{\approx} \boldsymbol{a}$ we establish the FE approximation $\mathbf{a}$ of $\boldsymbol{a}$ defined in the continuous model setting.

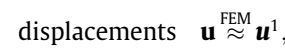



$$
\begin{aligned}
& \text { contact gap } \quad \mathbf{s}_{n} \stackrel{\text { FEM }}{\approx} s^{c}, \quad \text { at } y^{S} \in \Gamma_{c S}, \\
& \text { matrix of normals } \mathbf{N}_{n} \stackrel{\text { FEM }}{\approx} \boldsymbol{n}^{c}, \quad \text { at } y^{S} \in \Gamma_{c S}, \\
& \text { restriction - rotation matrix } \tilde{\mathbf{R}}_{n} \varphi=\mathbf{X}_{n} \mathbf{Q R} \varphi=\mathbf{N}_{n} \mathbf{R} \varphi \stackrel{\text { FEM }}{\approx} \boldsymbol{n}^{c} \cdot \boldsymbol{R} \varphi, \text { at } y^{S} \in \Gamma_{c S} \text {. }
\end{aligned}
$$


Let us recall that all the right hand side expressions depend on the local coordinates. In particular, $\mathbf{N}_{n}$ contains rows constituted by $\boldsymbol{n}^{c}$ at nodes of $\Gamma_{c s}$. In analogy with (6.3), stress $\mathbb{D} \boldsymbol{e}_{y}\left(\boldsymbol{G}^{0} \widehat{y}\right)$ induced by the macroscopic strain $\boldsymbol{G}^{0}$ is represented by

$\mathbf{g}=(\overline{\mathbf{K}} \overline{\mathbf{z}})^{\#}, \quad$ where $\left.\overline{\mathbf{z}}\right|_{\text {node }}=\left.\boldsymbol{G}^{0} \widehat{y}\right|_{\text {node }}$.

As before, $\tilde{\mathbf{u}}=\mathbf{Q u}$ is obtained by the rotation matrix which is now established for rotations of all DOFs on the entire contact boundary $\Gamma_{c}$. The residuum of the equilibrium associated with domain $Y_{S}$ is represented by

$$
\begin{aligned}
& \tilde{\mathbf{F}}(\tilde{\mathbf{u}})=\tilde{\mathbf{K}} \tilde{\mathbf{u}}+\tilde{\mathbf{g}}=\left[\tilde{\mathbf{F}}_{A} ; \tilde{\mathbf{F}}_{R} ; \tilde{\mathbf{F}}_{n S}\right], \quad \text { where } \\
& \tilde{\mathbf{F}}_{D}(\tilde{\mathbf{u}})=\mathbf{K}_{D} \tilde{\mathbf{u}}-\tilde{\mathbf{g}}_{D}, \quad D \in\{A, R, n S\} .
\end{aligned}
$$

By virtue of the RBM constraint related to the DOFs $\tilde{\boldsymbol{u}}_{R}$ on $\Gamma_{R S}$, (6.6) 1,2 applies in analogy with the treatment of (6.5). Thus, the residuals in $\tilde{\mathbf{F}}(\tilde{\mathbf{u}})$ are expressed explicitly, as follows:

$\tilde{\mathbf{F}}(\tilde{\mathbf{u}})=\left[\begin{array}{c}\tilde{\mathbf{F}}_{A} \\ \tilde{\mathbf{F}}_{R} \\ \tilde{\mathbf{F}}_{n S}\end{array}\right]=\left[\begin{array}{ccc}\tilde{\mathbf{K}}_{A A} & \tilde{\mathbf{K}}_{A R} \tilde{\mathbf{B}}_{R} & \tilde{\mathbf{K}}_{A n} \\ \tilde{\mathbf{K}}_{R A} & \tilde{\mathbf{K}}_{R R} \tilde{\mathbf{B}}_{R} & \tilde{\mathbf{K}}_{R n} \\ \tilde{\mathbf{K}}_{n A} & \tilde{\mathbf{K}}_{n R} \tilde{\mathbf{B}}_{R} & \tilde{\mathbf{K}}_{n n}\end{array}\right]\left[\begin{array}{c}\tilde{\mathbf{u}}_{A} \\ \mathbf{p} \\ \tilde{\mathbf{u}}_{n}\end{array}\right]+\left[\begin{array}{c}\tilde{\mathbf{g}}_{A}-\tilde{\mathbf{K}}_{A R} \tilde{\mathbf{z}}_{R} \\ \left(\tilde{\mathbf{g}}_{R}-\tilde{\mathbf{K}}_{R R} \tilde{\mathbf{z}}_{R}\right) \\ \tilde{\mathbf{g}}_{n}-\tilde{\mathbf{K}}_{n R} \tilde{\mathbf{z}}_{R}\end{array}\right]$,

recalling that $\mathbf{p}$ represents the rigid body modes of the inclusion. Now the contact problem can be defined.

Definition 4. Find $\left(\tilde{\mathbf{u}}_{A}, \tilde{\mathbf{u}}_{R}, \mathbf{p}, \tilde{\mathbf{u}}_{n S}\right)$ such that

$$
\begin{array}{ll}
\tilde{\mathbf{F}}_{A}(\tilde{\mathbf{u}}) & =0, \\
\tilde{\mathbf{B}}_{R}^{T} \tilde{\mathbf{F}}_{R}(\tilde{\mathbf{u}})-\tilde{\mathbf{B}}_{n}^{T} \tilde{\mathbf{F}}_{n S}(\tilde{\mathbf{u}}) & =0, \\
\tilde{\mathbf{H}}_{n}(\tilde{\mathbf{u}}) & =0, \\
\text { where } \tilde{\mathbf{H}}_{n}(\tilde{\mathbf{u}}) & :=\max \left\{\tilde{\mathbf{u}}_{n S}-\tilde{\mathbf{B}}_{n} \mathbf{p}-\hat{\mathbf{s}}_{n}(\mathbf{G}), \tilde{\mathbf{F}}_{n S}(\tilde{\mathbf{u}})\right\},
\end{array}
$$

$\tilde{\mathbf{F}}(\tilde{\mathbf{u}})$ is given in (6.12) and $\tilde{\mathbf{g}}$ is defined by (6.11).

This problem can be solved by the non-smooth Newton method. It can be seen easily that the third block of equations related to the normal-projected DOFs at the contact boundary $\Gamma_{n s}$ represents the complementarity problem (CP),

$$
\begin{array}{rll}
\text { kinematic constraints : } & \tilde{\mathbf{u}}_{n S}-\tilde{\mathbf{B}}_{n} \mathbf{p}-\hat{\mathbf{s}}_{n}(\mathbf{G}) & \leqslant 0, \\
\text { contact stress : } & \tilde{\mathbf{F}}_{n S}(\tilde{\mathbf{u}}) & \leqslant 0, \\
\text { complementarity : } & \left(\tilde{\mathbf{u}}_{n S}-\tilde{\mathbf{B}}_{n} \mathbf{p}-\hat{\mathbf{s}}_{n}(\mathbf{G})\right)^{T} \tilde{\mathbf{F}}_{n S}(\tilde{\mathbf{u}}) & =0,
\end{array}
$$

which in the FEM discretized forms corresponds to the $\mathrm{CP}$ imposed a.e. on $\Gamma_{c s}: \sigma_{n} \leqslant 0 \& u_{n}^{\text {rel }}-s \leqslant 0 \& \sigma_{n}\left(u_{n}^{\text {rel }}-s\right)=0$, where $u_{n}^{\text {rel }}$ is the normal-projected relative displacement at the homologous points, whereas $\sigma_{n}$ is the contact stress. The second block can be understood as the equilibrium of the rigid inclusion loaded on surfaces $\Gamma_{S R}$ and $\Gamma_{C R}$. In the next section, however, we show how (6.15) is derived from the FE-discretized variational inequality.

6.3. Relationship between the continuous and the discretized contact problem formulation

We show how the continuous formulation (5.6) is related to the discretized problem (6.14). Let us introduce the FE discretization of the displacement field extended by DOFs $\tilde{\mathbf{u}}_{n R}$ representing normal displacements at the homologous on $\Gamma_{c R}$,
$\tilde{\mathbf{u}}=\left[\begin{array}{c}\tilde{\mathbf{u}}_{A} \\ \tilde{\mathbf{u}}_{R} \\ \tilde{\mathbf{u}}_{n R} \\ \tilde{\mathbf{u}}_{n S}\end{array}\right]=\left[\begin{array}{ccc}\mathbf{I} & 0 & 0 \\ 0 & \tilde{\mathbf{B}}_{R} & 0 \\ 0 & \tilde{\mathbf{B}}_{n R} & 0 \\ 0 & 0 & \mathbf{I}\end{array}\right]\left[\begin{array}{c}\tilde{\mathbf{u}}_{A} \\ \mathbf{p} \\ \tilde{\mathbf{u}}_{n}\end{array}\right]$

where $\tilde{\mathbf{u}}_{n s}$ are related to the DOFs on $\Gamma_{c S}$. In analogy, one can consider discretized test displacement field $\tilde{\mathbf{v}}$ represented by $\left(\tilde{\mathbf{v}}_{A}, \mathbf{q}, \tilde{\mathbf{v}}_{n}\right)$. Further we define $\tilde{K}(\mathbf{G})=\left\{\tilde{\mathbf{v}} \mid \tilde{\mathbf{v}}_{n s}-\tilde{\mathbf{v}}_{n R}-\hat{\mathbf{s}}_{n}(\mathbf{G}) \leqslant 0\right\}$. Formal application of the FE-discretization transforms the variational inequality (5.6) into the following form,

$\tilde{\mathbf{u}} \in \tilde{K}(\mathbf{G}): \quad\langle\tilde{\mathbf{F}}(\tilde{\mathbf{u}}) \mid \tilde{\mathbf{v}}-\tilde{\mathbf{u}}\rangle \geqslant 0 \quad \forall \tilde{\mathbf{v}} \in \tilde{K}(\mathbf{G})$,

where $\tilde{\mathbf{F}}(\tilde{\mathbf{u}})$ has been introduced in (6.13) and $\langle\mathbf{a} \mid \mathbf{b}\rangle$ is the inner product of compatible vectors $\mathbf{a}, \mathbf{b} \in \mathbb{R}^{N}$, for any natural $N \geqslant 1$.

Proposition 2. Vector $\tilde{\mathbf{u}}$ solves (6.17) iff it satisfies (6.14).

To prove Proposition 2, we derive the nonsmooth equation directly form variational inequality (6.17). Without loss of generality, let the test vectors $\tilde{\mathbf{v}}_{n S}$ have the following form: $\tilde{\mathbf{v}}_{n S}:=\tilde{\mathbf{v}}_{n S}^{\prime}-\tilde{\mathbf{v}}_{n R}+\tilde{\mathbf{u}}_{n R}$. Note that any component of $\tilde{\mathbf{v}}_{n S}$ expresses displacement in the normal direction with respect to $\Gamma_{c R}$ at the homologous point of a point on $\Gamma_{c S}$, as well as the corresponding components of $\tilde{\mathbf{v}}_{n R}$ and $\tilde{\mathbf{u}}_{n R}$. Therefore, (6.17) can be written equivalently, as follows,

$$
\begin{aligned}
& \left\langle\tilde{\mathbf{F}}_{A}(\tilde{\mathbf{u}}) \mid \tilde{\mathbf{v}}_{A}-\tilde{\mathbf{u}}_{A}\right\rangle+\left\langle\tilde{\mathbf{F}}_{R}(\tilde{\mathbf{u}}) \mid \tilde{\mathbf{v}}_{R}-\tilde{\mathbf{u}}_{R}\right\rangle \\
& \quad+\left\langle\tilde{\mathbf{F}}_{n S}(\tilde{\mathbf{u}}) \mid \tilde{\mathbf{v}}_{n S}-\tilde{\mathbf{v}}_{n R}-\left(\tilde{\mathbf{u}}_{n S}-\tilde{\mathbf{u}}_{n R}\right)\right\rangle \geqslant 0,
\end{aligned}
$$

for all $\tilde{\mathbf{v}} \in \tilde{K}(\mathbf{G})$, where $\tilde{\mathbf{v}}_{n S}$ has been introduced above in terms of $\tilde{\mathbf{v}}_{n S}^{\prime}$. The last inner product in (6.18) can be split in two parts,

$\left\langle\tilde{\mathbf{F}}_{n S}(\tilde{\mathbf{u}}) \mid \tilde{\mathbf{v}}_{n S}-\tilde{\mathbf{u}}_{n S}\right\rangle-\left\langle\tilde{\mathbf{F}}_{n S}(\tilde{\mathbf{u}}) \mid \tilde{\mathbf{v}}_{n R}-\tilde{\mathbf{u}}_{n R}\right\rangle$.

Further we consider the rigid modes $\tilde{\mathbf{v}}_{n R}=\tilde{\mathbf{B}}_{n R} \mathbf{q}$ and $\tilde{\mathbf{u}}_{n R}=\tilde{\mathbf{B}}_{n R} \mathbf{p}$ with $\mathbf{q}$ and $\mathbf{p}$ independent of any restriction related to the contact condition. The restriction concerns the DOFs on the compliant surface $\Gamma_{c s}$ only. By the consequence, the convex set $\tilde{K}(\mathbf{G})$ presents the restriction for the solution part $\tilde{\mathbf{u}}_{n S}$ and the associated test modes $\tilde{\mathbf{v}}_{n s}$ only, being parameterized by the macroscopic strains $\mathbf{G}$ and the RBM modes $\mathbf{p}$, hence

$\tilde{K}(\mathbf{G}, \mathbf{p})=\left\{\tilde{\mathbf{v}}_{n S} \mid \tilde{\mathbf{v}}_{n S}-\tilde{\mathbf{B}}_{n R} \mathbf{p}-\hat{\mathbf{s}}_{n}(\mathbf{G}) \leqslant 0\right\}$.

Since the rigid modes are expressed by $\mathbf{p}$ and $\mathbf{q}$, by virtue of the split (6.19), inequality (6.18) is equivalent to

$$
\begin{aligned}
& \left\langle\tilde{\mathbf{F}}_{A}(\tilde{\mathbf{u}}) \mid \tilde{\mathbf{v}}_{A}-\tilde{\mathbf{u}}_{A}\right\rangle+\left\langle\tilde{\mathbf{F}}_{R}(\tilde{\mathbf{u}}) \mid \tilde{\mathbf{B}}_{R}(\mathbf{q}-\mathbf{p})\right\rangle-\left\langle\tilde{\mathbf{F}}_{n S}(\tilde{\mathbf{u}}) \mid \tilde{\mathbf{B}}_{n R}(\mathbf{q}-\mathbf{p})\right\rangle \\
& \quad+\left\langle\tilde{\mathbf{u}}_{n S}-\left[\tilde{\mathbf{u}}_{n S}-\tilde{\mathbf{F}}_{n S}(\tilde{\mathbf{u}})\right] \mid \tilde{\mathbf{v}}_{n S}-\tilde{\mathbf{u}}_{n S}\right\rangle \geqslant 0,
\end{aligned}
$$

for all $\tilde{\mathbf{v}}_{n S} \in \tilde{K}(\mathbf{G}, \mathbf{p})$ and for any $\tilde{\mathbf{v}}_{A}$ and $\mathbf{q}$. In the last inner product, upon adding and subtracting $\tilde{\mathbf{u}}_{n R}$ in both the arguments, we can define $\tilde{\mathbf{v}}_{n}:=\tilde{\mathbf{v}}_{n S}-\tilde{\mathbf{u}}_{n R}$ and $\tilde{\mathbf{u}}_{n}:=\tilde{\mathbf{u}}_{n S}-\tilde{\mathbf{u}}_{n R}$. This motivates us to introduce the convex set $\tilde{K}^{*}(\mathbf{G})=\left\{\mathbf{w}_{n} \mid \mathbf{w}_{n} \leqslant \hat{\mathbf{s}}_{n}(\mathbf{G})\right\}$, such that for a given $\mathbf{p}$, if $\tilde{\mathbf{v}}_{n S} \in \tilde{K}(\mathbf{G}, \mathbf{p})$, then also $\tilde{\mathbf{v}}_{n} \in \tilde{K}^{*}(\mathbf{G})$ and vice versa, recalling that $\tilde{\mathbf{u}}_{n R}:=\mathbf{B}_{n R} \mathbf{p}$. Since $\tilde{\mathbf{v}}_{A}$ and $\mathbf{q}$ are arbitrary, only the last inner product in (6.21) is subject to inequality, while the others must vanish. Therefore, (6.21) yields

$$
\begin{aligned}
\tilde{\mathbf{F}}_{A}(\tilde{\mathbf{u}}) & =0, \\
\tilde{\mathbf{B}}_{R}^{T} \tilde{\mathbf{F}}_{R}(\tilde{\mathbf{u}})-\tilde{\mathbf{B}}_{n R}^{T} \tilde{\mathbf{F}}_{n S}(\tilde{\mathbf{u}}) & =0, \\
\tilde{\mathbf{u}}_{n} \in \tilde{K}^{*}(\mathbf{G}), \quad\left\langle\tilde{\mathbf{u}}_{n}-\left[\tilde{\mathbf{u}}_{n}-\tilde{\mathbf{F}}_{n S}(\tilde{\mathbf{u}})\right] \mid \tilde{\mathbf{v}}_{n}-\tilde{\mathbf{u}}_{n}\right\rangle & \geqslant 0, \quad \forall \tilde{\mathbf{v}}_{n} \in \tilde{K}^{*}(\mathbf{G}) .
\end{aligned}
$$


Inequality in $(6.22)_{3}$ is equivalent to the projection

$\left\langle\mathbf{w}_{n}-\operatorname{Proj}_{K^{*}(G)}\left(\mathbf{w}_{n}-\tilde{\mathbf{F}}_{n S}(\tilde{\mathbf{u}})\right) \mid \tilde{\mathbf{v}}_{n}\right\rangle=0$,

where

$\operatorname{Proj}_{K^{*}(\boldsymbol{G})}(\mathbf{f})=\min \left\{\hat{\mathbf{s}}_{n}, \mathbf{f}\right\}=-\max \left\{-\hat{\mathbf{s}}_{n},-\mathbf{f}\right\}$,

therefore, using $\mathbf{f}:=\mathbf{w}_{n}-\tilde{\mathbf{F}}_{n S}(\tilde{\mathbf{u}})$, the left hand side in (6.23) is expressed by

$$
\begin{gathered}
\left\langle\mathbf{w}_{n}+\max \left\{-\hat{\mathbf{s}}_{n}, \tilde{\mathbf{F}}_{n S}(\tilde{\mathbf{u}})-\mathbf{w}_{n}\right\} \mid \tilde{\mathbf{v}}_{n}\right\rangle \\
=\left\langle\max \left\{\mathbf{w}_{n}-\hat{\mathbf{s}}_{n}, \tilde{\mathbf{F}}_{n S}(\tilde{\mathbf{u}})\right\} \mid \tilde{\mathbf{v}}_{n}\right\rangle .
\end{gathered}
$$

Recall that $\mathbf{w}_{n}=\tilde{\mathbf{v}}_{n s}-\tilde{\mathbf{u}}_{n R}$, hence (6.23) writes, as follows,

$\max \left\{\tilde{\mathbf{u}}_{n S}-\tilde{\mathbf{B}}_{n R} \mathbf{p}-\hat{\mathbf{s}}_{n}(\mathbf{G}), \tilde{\mathbf{F}}_{n S}(\tilde{\mathbf{u}})\right\}=0$.

which together with (6.22) completes the proof of (6.14).

\subsection{Algorithm of the semi-smooth Newton method}

To solve the local contact problem presented in the form of a non-smooth equation, we use the Semi-Smooth Newton (SSN) method proposed in [8] and applied successfully to solve contact problems of elastic, or elasto-plastic solids [25]. Since this approach to solving the contact problems is not standard in the computational mechanics community, for the paper completeness, here we present the main idea of the semi-smooth method which we employ in Section 7, where few numerical illustrations are shown.

Problem (6.14) can be presented in an abstract form: Find $x \in \mathbb{R}^{N}$, such that

$\max \{\mathrm{A}(\mathrm{x}), \mathrm{B}(\mathrm{x})\}=0$,

where functions $A(x), B(x) \in \mathbb{R}^{N}$ are differentiable with respect to $x$; in fact, due to the structure of problem (6.14), functions $A(x), B(x)$ are linear in $\mathrm{x}$, since the macro-strain $\boldsymbol{G}$ is assumed to be given when solving the local problem. Recall that (6.27) is understood componentwise, i.e. $\max \left\{A_{i}, B_{i}\right\}=0$ for all $i=1, \ldots, N$, which is equivalent to the complementarity problem $A_{i} \leqslant 0, B_{i} \leqslant 0$, and $A_{i} B_{i}=0$. Obviously, the form (6.27) of the non-smooth equation is relevant for the block $\tilde{\mathbf{H}}_{n}$ involved in (6.14). However, also the standard block constituting this problem can be presented in the form (6.27), whereby the associated components are considered identical, thus, $A_{i} \equiv B_{i}$ for the non-contact DOFs $i$.

An efficient semi-smooth Newton method solver is based on a reformulation of problem (6.27) in terms of the Fischer-Burmeister function $\boldsymbol{\Phi}(\mathrm{x})=\left(\Phi_{i}(\mathrm{x})\right)$, and the merit function $\Psi(\mathrm{x})$, where

$$
\begin{aligned}
& \Phi_{i}=\sqrt{A_{i}^{2}+B_{i}^{2}}+A_{i}+B_{i}, i=1, \ldots, N, \\
& \Psi(\mathrm{x})=\frac{1}{2}\|\boldsymbol{\Phi}(\mathrm{x})\|^{2}=\frac{1}{2} \sum_{i=1}^{N}\left(\Phi_{i}(\mathrm{x})\right)^{2} .
\end{aligned}
$$

Solutions of Problem (6.27) are equivalently defined, as follows:

$\Phi(\mathrm{x})=0 \Longleftrightarrow \min _{\mathrm{x} \in \mathbb{R}^{N}} \Psi(\mathrm{x})$.

The second alternative of Problem (6.29) leads to construction of a globally convergent algorithm. It is worth to note that $\Phi_{i}$ is differentiable everywhere but in the origin, i.e. only subdifferential is defined whenever $A_{j}=B_{j}=0$ for some $j$. Let $Z_{\boldsymbol{\Phi}}=\left\{x \in \mathbb{R}^{N} \mid \exists j\right.$ : $\left.A_{j}(\mathrm{x})=B_{j}(\mathrm{x})=0\right\}$. Further we introduce subset $D_{\Phi} \subset \mathbb{R}^{N}$ containing all points where $\boldsymbol{\Phi}$ is differentiable. Obviously, $D_{\Phi}=\mathbb{R}^{N} \backslash Z_{\boldsymbol{\Phi}}$. The algorithm, as introduced below according to [8] and implemented to solve (6.27), uses through the notion of the B-differentiability, cf. [21,7]. In particular, the B-subdifferential of $\boldsymbol{\Phi}$ at $\hat{x}$, denoted by $\partial_{B} \boldsymbol{\Phi}(\hat{\mathbf{x}})$, is defined in terms of sequence of gradients $\nabla \boldsymbol{\Phi}\left(x^{j}\right)$ over a convergent sequence $\left\{x^{j}\right\}$ of points approximating $\mathrm{x}$,

$\partial_{B} \boldsymbol{\Phi}(\hat{\mathrm{x}})=\left\{\boldsymbol{\Xi} \in \mathbb{R}^{N} \times \mathbb{R}^{N} \mid \exists\left\{x^{j}\right\}, x^{j} \in D_{\boldsymbol{\Phi}}, \boldsymbol{\Xi}=\lim _{x^{j} \rightarrow \hat{\mathrm{x}}} \nabla \boldsymbol{\Phi}\left(x^{j}\right)\right\}$.

Algorithm Alg-SSN. (Solutions of the local contact problem). Set parameters: $\rho>0, p>2, \epsilon>0$, and $\beta \in] 0,1 / 2]$;

(0) $k:=0$ and define $x^{k} \in \mathbb{R}^{N}$;

(1) If $\Psi\left(x^{k}\right)\left\|\nabla \Psi\left(x^{k}\right)\right\| \leqslant \epsilon$, then STOP.

(2) Define subgradient (gradient) $\mathcal{G}^{k} \in \partial_{B} \boldsymbol{\Phi}\left(x^{k}\right)$, (a) If $\mathcal{G}^{k}$ is a regular matrix, compute $d^{k} \in \mathbb{R}^{N}$, such that $\mathcal{G}^{k} \mathrm{~d}^{k}=-\boldsymbol{\Phi}\left(\mathrm{x}^{k}\right)$

and verify "a sufficient" decrease of the descent step,

$\nabla \Psi\left(x^{k}\right) \cdot d^{k} \leqslant-\rho\left\|d^{k}\right\|^{p}$

(b) If $d^{k}$ is not defined in step 2a), or if (6.32) fails, define $\mathrm{d}^{k}:=-\nabla \Psi\left(\mathrm{x}^{k}\right)$.

(3) Define new approximation $x^{k+1}$ using the Linesearch procedure, i.e. find maximum $t \in] 0,1]$, such that $\Psi\left(\tilde{x}^{k}\right) \leqslant \tilde{\Psi}^{k}$, where $\tilde{x}^{k}:=x^{k}+t d^{k}$ and $\tilde{\Psi}^{k}:=\Psi\left(x^{k}\right)+\beta t \nabla \Psi\left(x^{k}\right) \cdot d^{k}$.

(4) Set $x^{k+1}:=\tilde{x}^{k}$ and $k:=k+1$; GOTO step 1 ).

In step (2), the subgradient $\mathcal{G}^{k}$ is computed in accordance with the definition of the subdifferential (6.30). It is worth to remark, that in most cases, functions $\Phi_{i}$ are differentiable, i.e. $A_{i} \neq B_{i}$. Let us define the set of semiactive contact nodes $I_{0}=\left\{i \in 1, \ldots, N \mid A_{i}=B_{i}=0\right\}$. Now $\mathcal{G}$ at a point $\times$ is computed, as follows: Let $\mathcal{G}_{i}$ denote the $i$-the row of matrix $\mathcal{G}$. Define a vector $z \in \mathbb{R}^{N}$, such that $z_{k} \neq 0$ iff $k \in I_{0}$, then

$$
\begin{aligned}
\text { for } i \notin I_{0}: \quad \mathcal{G}_{i}= & \left(\frac{A_{i}(\mathrm{x})}{\sqrt{\left(A_{i}(\mathrm{x})\right)^{2}+\left(B_{i}(\mathrm{x})\right)^{2}}}+1\right) \nabla A_{i}(\mathrm{x}) \\
& +\left(\frac{B_{i}(\mathrm{x})}{\left.\sqrt{\left(A_{i}(\mathrm{x})\right)^{2}+\left(B_{i}(\mathrm{x})\right)^{2}}+1\right) \nabla B_{i}(\mathrm{x}),}\right. \\
\text { for } i \in I_{0}: \quad \mathcal{G}_{i}= & \left(\frac{\mathrm{z} \cdot \nabla A_{i}(\mathrm{x})}{\sqrt{\left(\mathrm{z} \cdot \nabla A_{i}(\mathrm{x})\right)^{2}+\left(\mathrm{z} \cdot \nabla B_{i}(\mathrm{x})\right)^{2}}}+1\right) \nabla A_{i}(\mathrm{x}) \\
& +\left(\frac{\mathrm{z} \cdot \nabla B_{i}(\mathrm{x})}{\sqrt{\left(\mathrm{z} \cdot \nabla A_{i}(\mathrm{x})\right)^{2}+\left(\mathrm{z} \cdot \nabla B_{i}(\mathrm{x})\right)^{2}}}+1\right) \nabla B_{i}(\mathrm{x}) .
\end{aligned}
$$

To explain briefly formula (6.33) $)_{2}$, for $i \in I_{0}$ the subgradient $\mathcal{G}_{i}$ is calculated using perturbed function $\tilde{\Phi}_{i}$ expressed in terms of $\tilde{A}_{i}$ and $\tilde{B}_{i}$ which are defined as functions of parameter $t \geqslant 0$ by $\tilde{A}_{i}(t):=A_{i}(\mathrm{x})+t z \cdot$ (in analogy for $\tilde{B}_{i}(t)$ ), correspondingly to the perturbed position $\tilde{x}=\mathrm{x}+t \mathrm{z}$. Then $\mathcal{G}_{i} \cdot \mathrm{z}=\lim _{t \rightarrow 0} \tilde{\Phi}_{i}(t)$ is the derivative in direction z. This proof assumes differentiability of both $A_{i}$ and $B_{i}$ which holds due to the specific problem treated here.

\section{Numerical examples}

In this section, we illustrate the numerical simulation of the selfcontact in the deforming porous medium. Examples which are dis- 

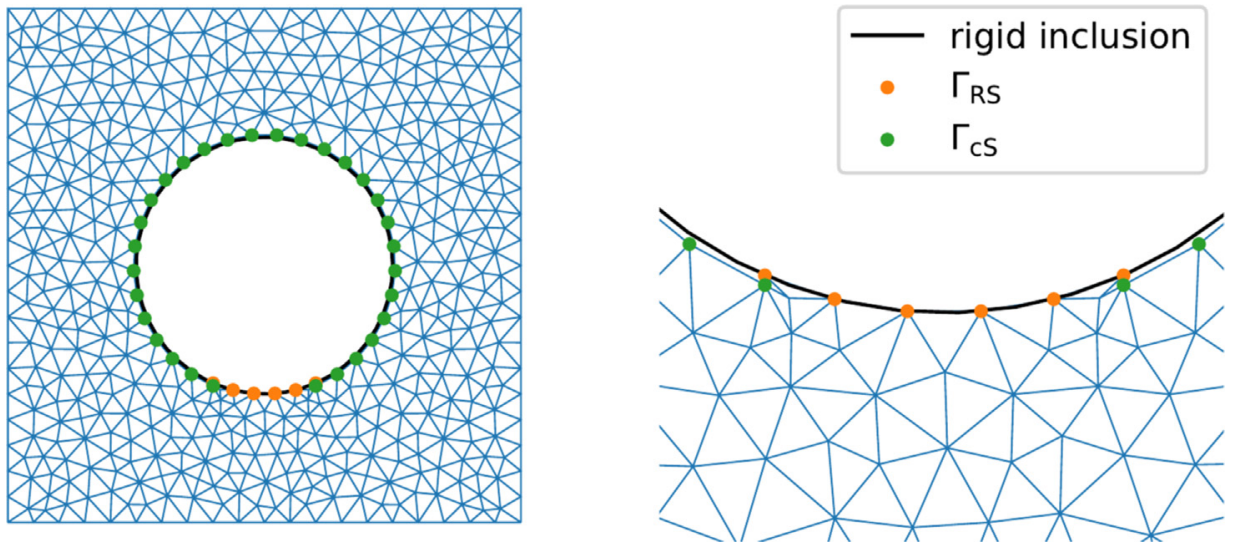

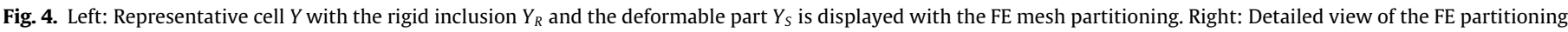
near the interface $\Gamma_{R S}$ and the contact gap $s$.

cussed below involve a 2D elastic structure with the periodically distributed rigid circular inclusions. As the consequence of the dimension restriction and the inclusions rigidity, plane strain problems are considered. The material properties are given by the Young modulus, $E=1 \mathrm{~Pa}$, and the Poisson ratio, $v=0.3$. In Fig. 4, the compliant part $Y_{m}$ of representative cell $\left.Y=\right]-1,1[\times]-1,1[$ is partitioned by the triangular finite element mesh, so that P1 polynomial approximation of the displacements is used. The inclusion $Y_{R}$ is clamped in the elastic matrix on a short interface $\Gamma_{R S}$, see Figs. 3 and 4. As the consequence of this geometric layout, the microstructure is symmetric with respect to the $y_{2}$ axis only. The geometric non-symmetry with respect to the $y_{1}$ axis leads to different microscopic responses while the same loading is applied subsequently in one, or the other axis, as will be demonstrated in Section 7.1. An influence of this non-symmetry feature inducing anisotropy of the homogenized medium will be discussed below, namely in Section 7.2, in the context of two uniaxial loading tests.

\subsection{Microscopic response with prescribed macroscopic strains}

The local contact problem (6.15) with given $\boldsymbol{G}=\boldsymbol{e}_{x}\left(\boldsymbol{u}^{0}\right)$ is solved for two loading cases with prescribed macroscopic compression strains:
- Case 1: compression in $x_{1}$-axis,

$$
\boldsymbol{e}_{x}\left(\boldsymbol{u}^{0}\right)=\left[\begin{array}{cc}
-0.2 & 0 \\
0 & 0.04
\end{array}\right]
$$

- Case 2: compression in $x_{2}$-axis,

$$
\boldsymbol{e}_{x}\left(\boldsymbol{u}^{0}\right)=\left[\begin{array}{cc}
0.04 & 0 \\
0 & -0.2
\end{array}\right]
$$

The solutions are displayed in terms of the stress distribution and the contact pressures on the "true" contact boundary $\Gamma_{c S}^{*}$ in Figs. 5 and 6 . We recall the microstructure non-symmetry which leads to quite different distributions of the contact nodes and contact pressures, when the Case 1 response is compared with the one of the Case 2.

In both cases, only 4 iterations of the semi-smooth Newton solver, as introduced in Section 6.4, were needed to achieve the precision $\Psi \leqslant 10^{-7}$, see Fig. 7 .

\subsection{Two scale response with prescribed macroscopic loads}

We present two examples illustrating a macroscopic uniaxial tension of a strip and a bending of a short cantilever beam. In both stress, $\sigma_{11}$

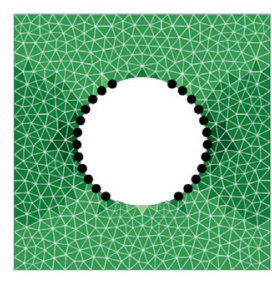

contact tractions

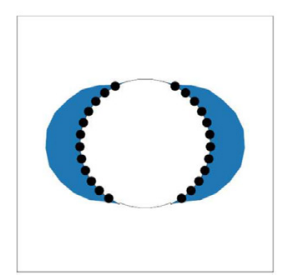

stress, $\sigma_{12}$

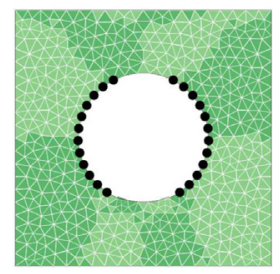

stress, $\sigma_{22}$

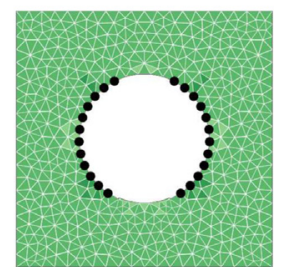



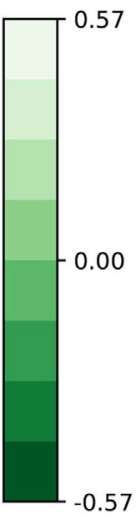

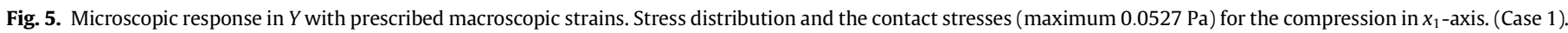


stress, $\sigma_{11}$



contact tractions

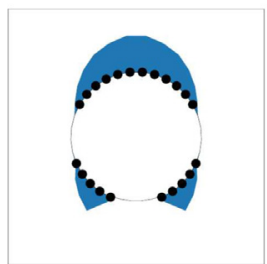

stress, $\sigma_{12}$

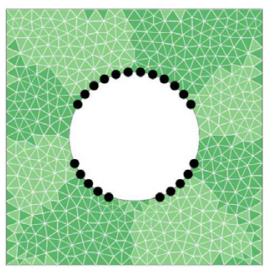

stress, $\sigma_{22}$

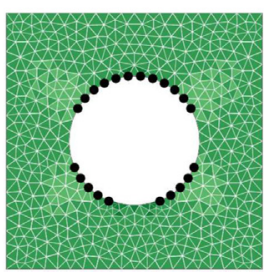

- $\Gamma_{\mathrm{cS}}^{*}$

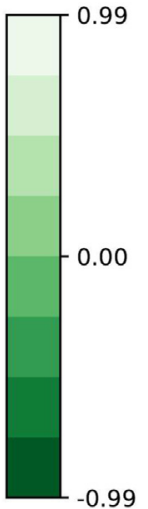

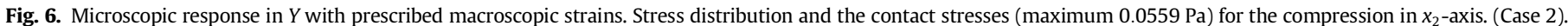

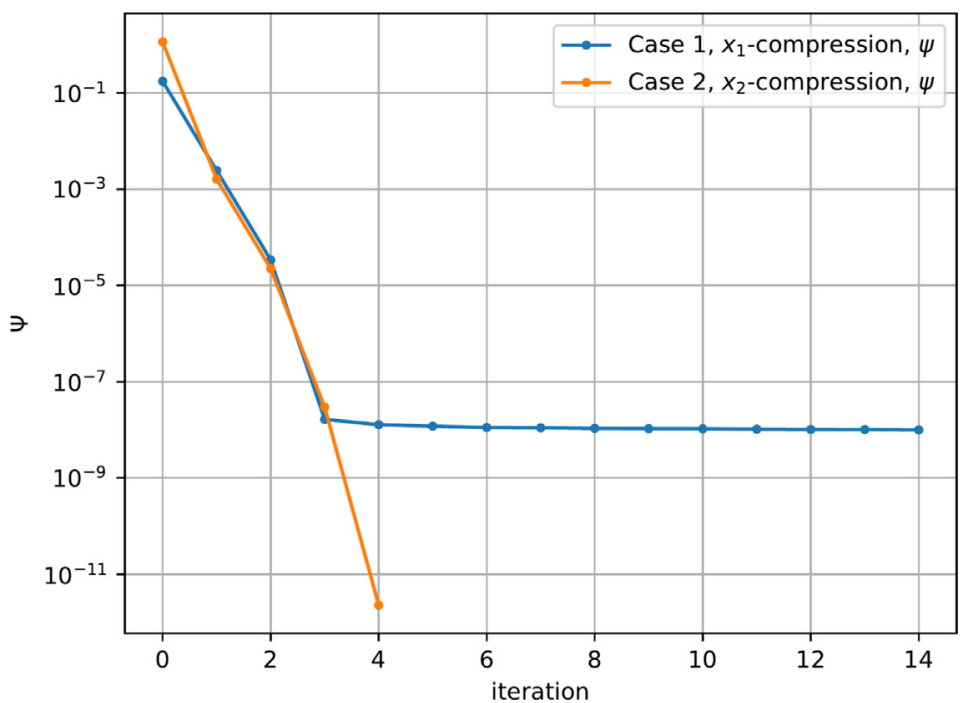

Fig. 7. Convergence of the SSN Algorithm, decrease of the merit function $\Psi$ for the two test cases with strains prescribed by (7.1) and (7.2),

these examples, the porous body occupies domain $\Omega=[0, L] \times[0, L]$, where $L=1$, and its periodic structure is generated by the representative cell described above. The discretized macroscopic model of the homogenized porous material comprises $M_{e}=908$ P1 elements yielding approximately $N_{\text {dof }}=512$ DOFs describing the in-plane displacement field. The homogenized consistent elastic stiffness tensors reflect the plane strain restrictions applied at the micro-level. By $\Gamma_{\sigma}$ and $\Gamma_{u}$ we refer to the parts of the boundary $\partial \boldsymbol{\Omega}$, where the traction loads and the displacements are prescribed, respectively, whereby $\Gamma_{u} \subset \partial_{u} \Omega$ and $\Gamma_{\sigma} \subset \partial_{\sigma} \Omega$ in the context of the boundary decomposition (2.2). The Global Two-scale Algorithm (Alg-G) introduced in Section 4 is implemented with the loads applied in a single increment, thus, the reference configuration is the initial, undeformed one. For both the stop-criteria in (4.17), the tolerance was $\epsilon_{1}=\epsilon_{2}=10^{-8}$.

\subsubsection{Uniaxial tension/compression in the axis of symmetry}

The tension strip is loaded by evenly distributed traction stress aligned with $x_{2}$-axis direction. Correspondingly, the boundary segments are defined, as follows:

$$
\Gamma_{u}=\left\{x \in \partial \Omega \mid x_{2}=0\right\}, \quad \Gamma_{\sigma}=\left\{x \in \partial \Omega \mid x_{2}=L\right\} .
$$

The uniform traction stress $\boldsymbol{b}=\left(0, \bar{b}_{2}\right)$ is prescribed on $\Gamma_{\sigma}$ with varying load $\bar{b}_{2} \in[-\bar{\sigma},+\bar{\sigma}]$, where $\bar{\sigma}=0.2 \mathrm{~Pa}$. The vertical displacements vanish (thus $u_{2}=0$ ) on $\Gamma_{u}$. For uniqueness of the solutions, we fix the structure at $\bar{x}=(0,0)$, thus, $\boldsymbol{u}(\bar{x})=\mathbf{0}$.

In Fig. 8, the macroscopic configuration is depicted for the minimum and maximum applied loading stress. Due to the loading aligned with the orthotropy axis, the strains are distributed uniformly in the whole of $\Omega$. The uniaxial stress-strain dependence is captured in Fig. 9(a), whereas the associated effective stiffness is displayed in terms of the components in Fig. 9(b). It shows the variable elasticity of the effective homogenized medium with progressive stiffening for compressive loads, although the stiffness increases also for some tensile loads. In order to illustrate the convergence properties of the proposed Algorithm Alg-G, the equilibrium residual (the out-of-balance) and the solution correction involved in the stopping criteria (4.17) are reported in Fig. 10. The Global Two-scale Algorithm terminates usually after 10-30 iterations labelled by $i$ the Algorithm Alg-G, see Fig. 11. However, 


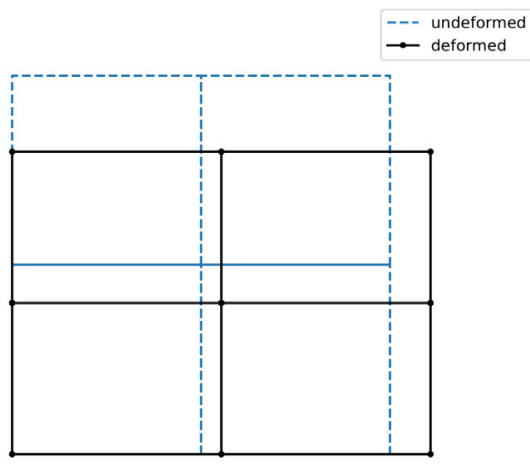

(a) compression $\bar{\sigma}=-0.2 \mathrm{~Pa}$

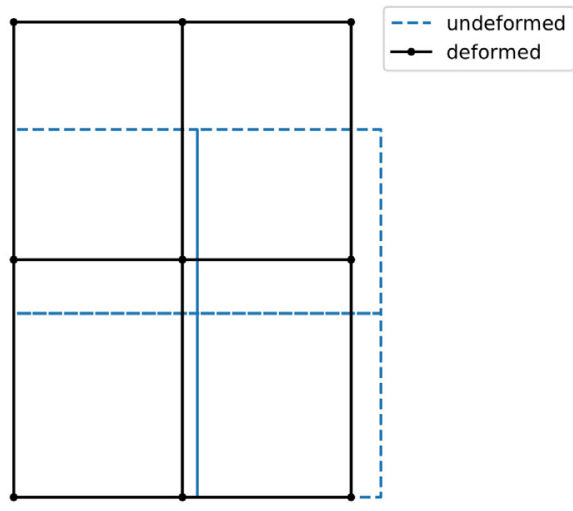

(b) tension $\bar{\sigma}=0.2 \mathrm{~Pa}$

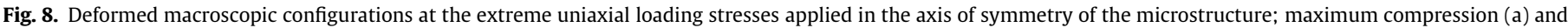
tension (b).

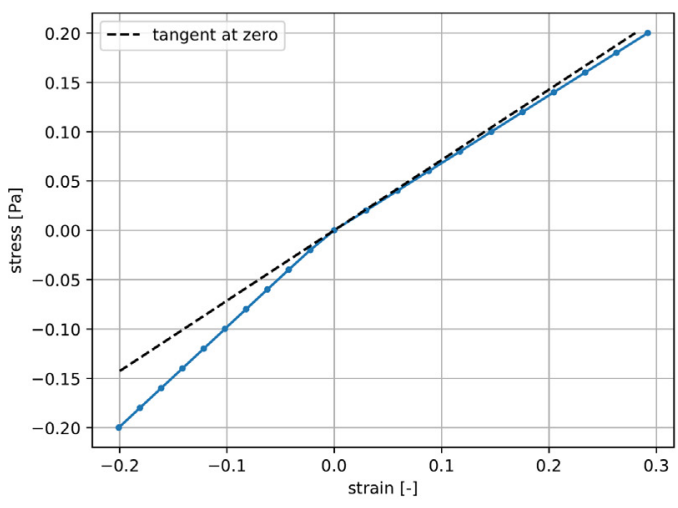

(a)



(b)

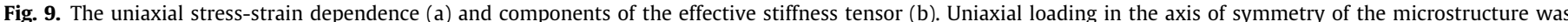
applied.

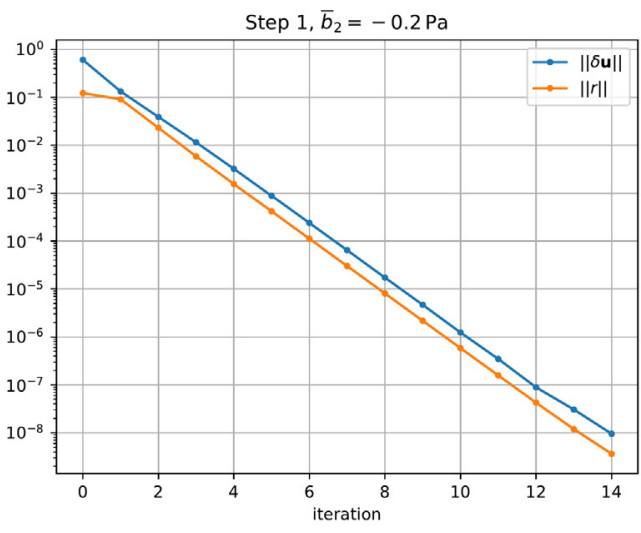

(a)

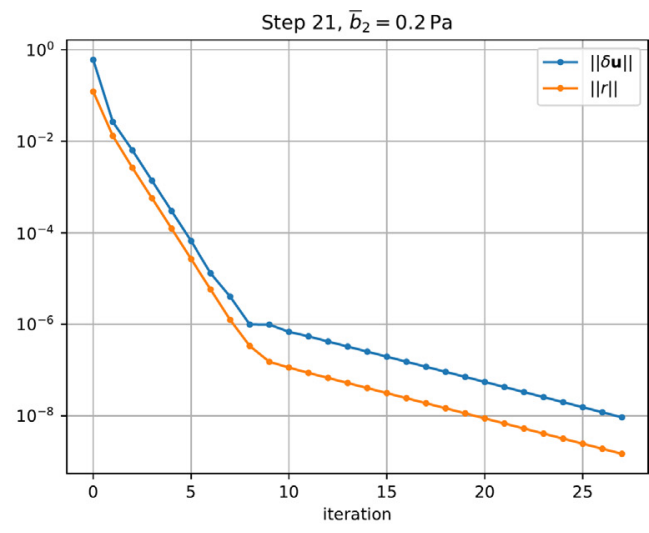

(b)

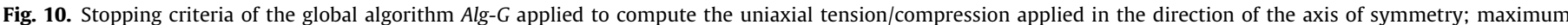
compression (a), maximum tension (b). 


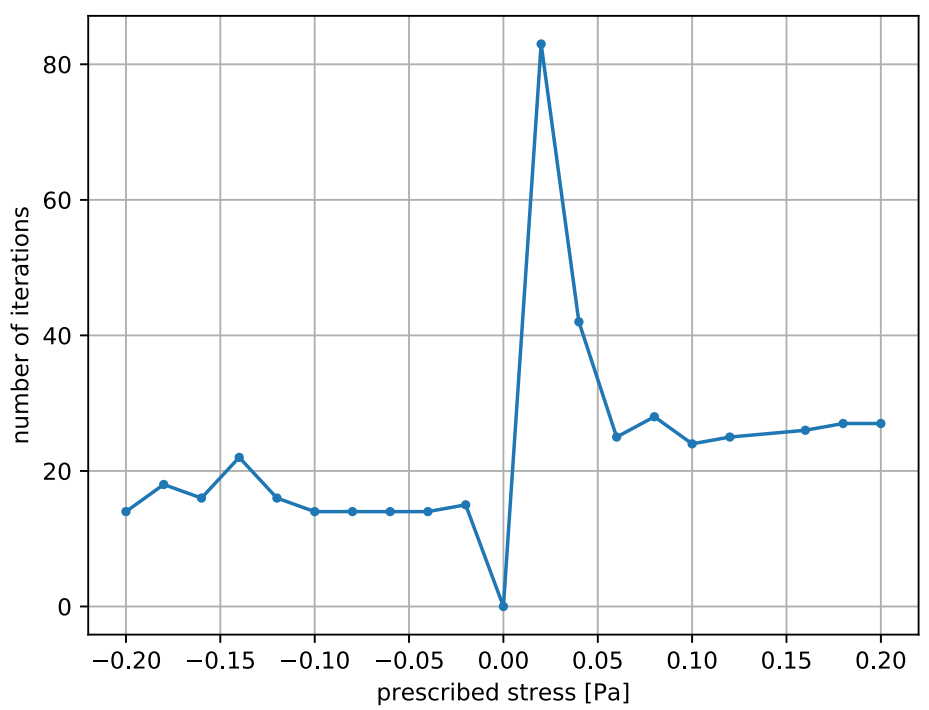

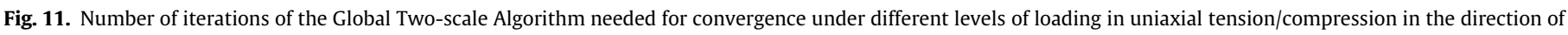
the axis of symmetry.

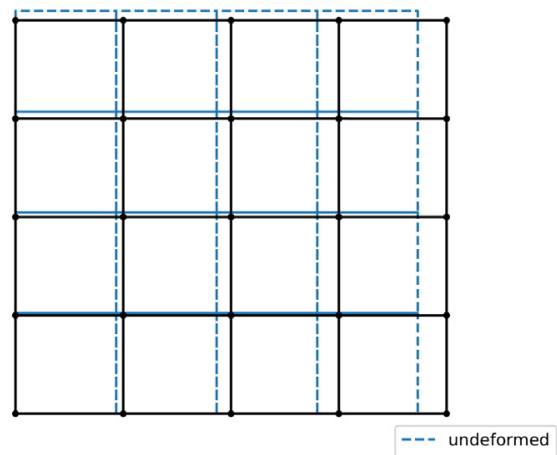

deformed



$\rightarrow$ undeformed

(a) tension $\bar{\sigma}=0.05 \mathrm{~Pa}$

(b) compression $\bar{\sigma}=-0.05 \mathrm{~Pa}$

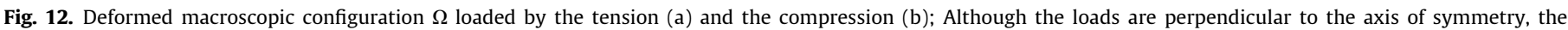
macroscopic responses are homogeneous in $\Omega$.

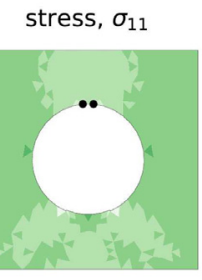

contact tractions



stress, $\sigma_{12}$

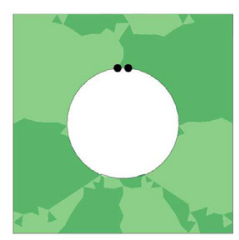

stress, $\sigma_{22}$

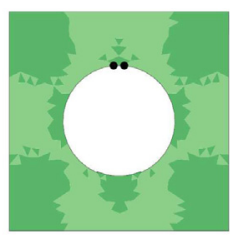

- $\Gamma_{\mathrm{cS}}^{*}$

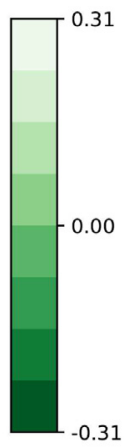

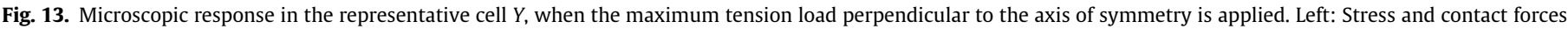
distributions; the maximum contact stress is $3.38 \cdot 10^{-3}$ Pa. Right: The deformed cell $Y$ at the micro-level (FE mesh displayed). 



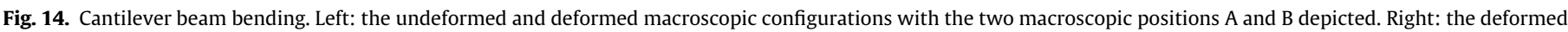
configuration with the distribution of the strain norm (mean values over finite elements).

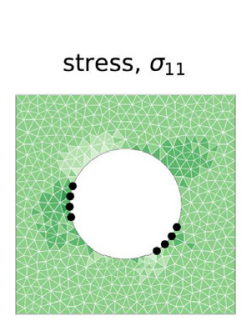

contact tractions

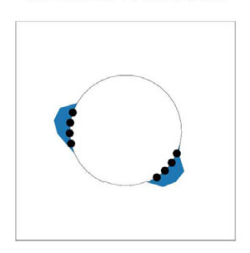

stress, $\sigma_{12}$

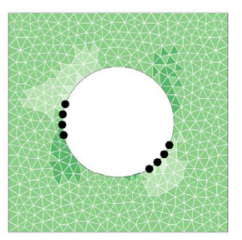

stress, $\sigma_{22}$



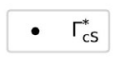

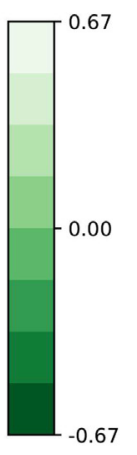

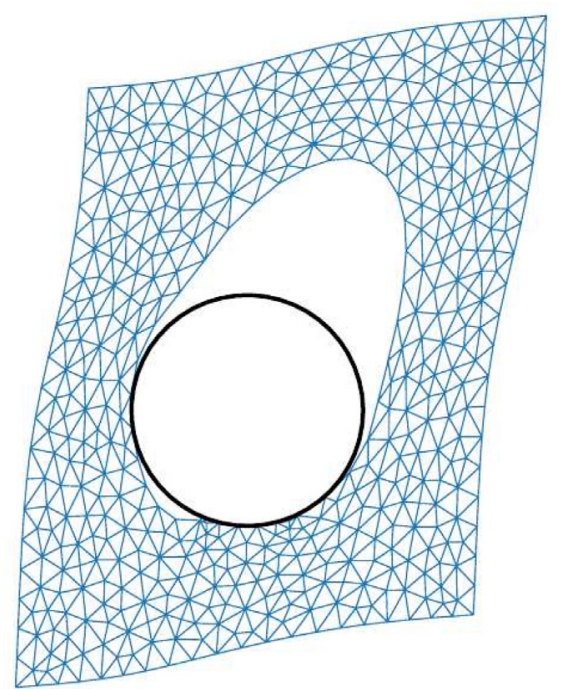

macroscopic position A, maximum contact stress $0.0292 \mathrm{~Pa}$

stress, $\sigma_{11}$

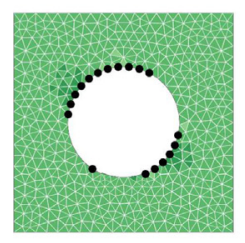

contact tractions

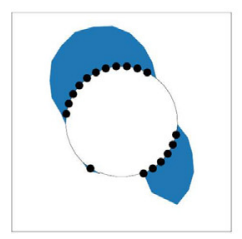

stress, $\sigma_{12}$

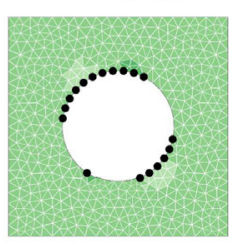

stress, $\sigma_{22}$

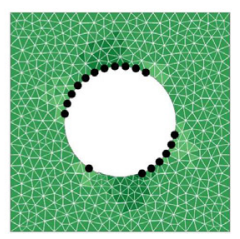

- $\Gamma_{c s}^{*}$

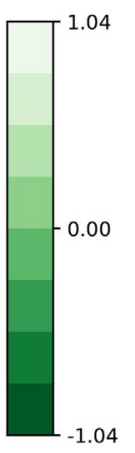



macroscopic position $\mathrm{B}$, maximum contact stress $0.0808 \mathrm{~Pa}$

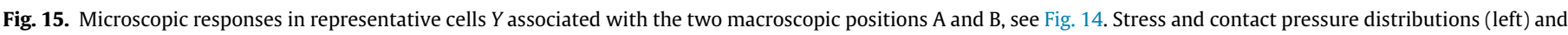
the deformed microconfigurations (right). 



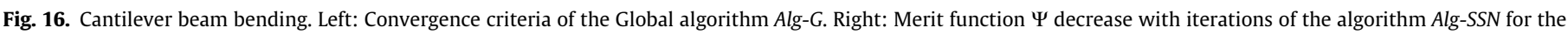
two macroscopic positions A and B.

a significant increase of iterations appears for small tension loads; this may be explained by semiactive constraints and by the observation that the current contact zone $\Gamma_{*}^{i}$ varies remarkably.

\subsubsection{Uniaxial tension/compression perpendicular to the axis of symmetry}

The reported simulation examines the influence of the transversal stiffening of the effective material induced by the uniaxial tension of the strip. The evenly distributed tractions are aligned with $x_{1}$-axis direction, so that the boundary segments are defined, as follows:

$$
\Gamma_{u}=\left\{x \in \partial \Omega \mid x_{1}=0\right\}, \quad \Gamma_{\sigma}=\left\{x \in \partial \Omega \mid x_{1}=L\right\} .
$$

The loads are presented by the uniform traction stress $\boldsymbol{b}=\left(\bar{b}_{1}, 0\right)$ prescribed on $\Gamma_{\sigma}$ with $\bar{b}_{1} \in[-\bar{\sigma},+\bar{\sigma}]$ with $\bar{\sigma}=0.05 \mathrm{~Pa}$, whereby the structure is fixed at $\bar{x}=(0,0)$, thus, $\boldsymbol{u}(\bar{x})=\mathbf{0}$.

The deformed macroscopic configuration is displayed in Fig. 12 for the maximum applied tension and compression loads. The macroscopic deformation remained homogeneous through the whole strip. The deformed state at the level of the porosity is illustrated in Fig. 13, where stresses in the local representative cells are reported. To solve the local contact problems, less than 6 iterations of the Alg-SSN were needed. The global algorithm Alg-G converged in between 10 and 70 iterations. As an exception to this behavior, for the loading step 5 with traction $\bar{b}_{1}=0.0166 \mathrm{~Pa}$, the global algorithm Alg-G converged only for the precision $\approx 10^{-7}$.

\subsubsection{Cantilever beam bending}

A short cantilever beam represented by domain $\Omega$ is clamped on its bottom edge $\Gamma_{u}=\left\{x \in \partial \Omega \mid x_{2}=0\right\}$, where the Dirichlet boundary conditions are prescribed, $\boldsymbol{u}=\mathbf{0}$. The beam is loaded by horizontal uniform traction stress $\boldsymbol{b}=\left(\bar{b}_{1}, 0\right)$ with $\bar{b}_{1}=0.05$ Pa acting on its top horizontal edge $\Gamma_{\sigma}=\left\{x \in \partial \Omega \mid x_{2}=L\right\}$.

As in the previous example, we report the deformed macroscopic configurations and the strain distribution in Fig. 14. The deformed state at the level of the porosity is illustrated in Fig. 15 in terms of solutions of the local contact problems. Also in this test, the convergence rates and the number of iterations of both the algorithms Alg- $\mathrm{G}$ and $\mathrm{Alg}$-SSN reach the same figures; in particular, less than 6 iterations are needed to decrease the merit function so that it attains values $\Psi<10^{-7}$, see Fig. 16).

\section{Conclusion}

We presented the two-scale limit model of periodic porous elastic medium with unilateral contact conditions prescribed on subparts of its pores. This nonlinear model involves the global equilibrium equation with the stress and stiffness tensors depending on solutions of the local contact problems, each being associated with the local microconfiguration which deforms according to the local macroscopic strain. Since the local problems are highly nonlinear because of the contact interaction, the "decoupling procedure" applicable in linear problems, cannot be pursued here: the two scale displacement field describing the local fluctuations of order $\varepsilon$ cannot be expressed in terms of the so-called characteristic autonomous responses (solutions of the corrector problems). However, due to the consistent linearization, the decoupling procedure can be applied to compute local effective stiffness tensors corresponding to the current deformation state.

To solve the two-scale problem numerically, an iterative algorithm with the consistent macroscopic tangent elastic modulus has been implemented for structures with rigid inclusions. Its performance has been tested on a number of 2D examples. The local contact problems are formulated in terms of nonsmooth equations which are solved by the semi-smooth Newton method based on the complementarity problem reformulation using the FischerBurmeister function. The numerical tests have shown very good performance of this method, especially when compared to usual non-smooth Newton methods. The convergence at the macroscopic level deteriorates if the "true", i.e. the actual contact zone moves significantly along the contact boundary and the so-called semiactive contact conditions appear. For this situation, further improvements of the proposed algorithms are being tested and will be reported in a separate study.

As a further extension of the nonlinear two-scale poroelastic model, an advanced contact interaction, taking into account the friction on the contact surface, or the interaction with the pore fluid, are studied in the context of the numerical approach reported in this paper. Moreover, the model can be extended for the treatment of the "symmetric self-contact", see the related algorithms $[12,19]$, taking into account completely compliant microstructure and describing the finite deformation. For the latter modelling feature, an approximation of the local responses can be established using the sensitivity analysis approach [23], cf. [24]. It allows to reduce the number of the representative micro-configurations where the local contact problems must be solved to cover the macroscopic configuration by the local effective stiffness tensors.

\section{Acknowledgment}

This research was supported by project GACR 19-04956S of the Scientific Foundation of the Czech Republic and due to the European Regional Development Fund-Project "Application of Modern Technologies in Medicine and Industry" (No. CZ.02.1.01/0.0/ 
0.0/17 048/0007280), and in part by project LO 1506 of the Czech Ministry of Education, Youth and Sports.

\section{References}

[1] Argatov II, Mel'nyk TA. Homogenization of a contact problem for a system of densely situated punches. Euro J Mech - A/Solids 2001;20(1):91-8.

[2] Brands B, Davydov D, Mergheim J, Steinmann P. Reduced-order modelling and homogenisation in magneto-mechanics: a numerical comparison of established hyper-reduction methods. Math Comput Appl 2019;24(1).

[3] Capatina A, Ene H, Timofte C. Homogenization results for elliptic problems in periodically perforated domains with mixed-type boundary conditions. Asympt Anal 2012;80:45-56.

[4] Chu A, Du S, Su Y. A new smoothing Conjugate Gradient Method for solving nonlinear nonsmooth complementarity problems. Algorithms 2015;8 (4):1195-209.

[5] Cioranescu D, Damlamian A, Griso G. The periodic unfolding method in homogenization. SIAM J Math Anal 2008;40(4):1585-620.

[6] Cioranescu D, Damlamian A, Orlik J. Homogenization via unfolding in periodic elasticity with contact on closed and open cracks. Asympt Anal 2013;82(34):201-32.

[7] Clarke FH. Optimization and nonsmooth analysis. Classics in applied mathematics. Soc Ind Appl Math 1990.

[8] De Luca T, Facchinei F, Kanzow C. A semismooth equation approach to the solution of nonlinear complementarity problems. Math Program 1996;75 (3):407-39.

9] Drosopoulos GA, Wriggers P, Stavroulakis GE. A multi-scale computational method including contact for the analysis of damage in composite materials. Comput Mater Sci 2014;95:522-35.

[10] Du S, Ben H. Dhia. An asymptotic numerical method to solve compliant Lennard-Jones-based contact problems involving adhesive instabilities. Comput Mech 2018.

[11] Fritzen F, Marfia S, Sepe V. Reduced order modeling in nonlinear homogenization: a comparative study. Comput Struct 2015;157:114-31.

[12] Gabriel D, Plešek J, Ulbin M. Symmetry preserving algorithm for large displacement frictionless contact by the pre-discretization penalty method. Int J Numer Meth Eng 2004;61(15):2615-38.

[13] Gilbert RP, Panchenko A, Xie X. Homogenization of a viscoelastic matrix in linear frictional contact. Math Meth Appl Sci 2004;28(3):309-28.
[14] Greco F. A study of stability and bifurcation in micro-cracked periodic elastic composites including self-contact. Int J Solids Struct 2013;50(10):1646-63.

[15] Griso G, Migunova A, Orlik J. Homogenization via unfolding in periodic layer with contact. Asympt Anal 2016;99(1-2):23-52.

[16] Haslinger J, Hlaváček I, Nečas J. Numerical methods for unilateral problems in solid mechanics. In: Finite element methods (Part 2), numerical methods for solids (Part 2). Handbook of numerical analysis, vol. 4. Elsevier; 1996. p. 313-485.

[17] Haslinger J, Miettinen M, Panagiotopoulos PD. Finite element method for hemivariational inequalities: theory, methods and applications. Nonconvex optimization and its applications. Springer US; 2010.

[18] Hlaváček I, Haslinger J, Nečas J, Lovíšek J. Solution of variational inequalities in mechanics. Applied mathematical sciences. Springer; 1988.

[19] Kopačka J, Tkachuk A, Gabriel D, Kolman R, Bischoff M, Plešek J. On stability and reflection-transmission analysis of the bipenalty method in contactimpact problems: a one-dimensional, homogeneous case study. Int J Numer Meth Eng 2018;113(10):1607-29.

[20] Mikelić A, Shillor M, Tapiéro R. Homogenization of an elastic material with inclusions in frictionless contact. Math Comput Model 1998;28(4):287-307. Recent advances in contact mechanics.

[21] Robinson SM. Nonlinear analysis and optimization. Mathematical programming studies, chapter Local structure of feasible sets in nonlinear programming, Part III: Stability and sensitivity, vol. 30. Berlin, Heidelberg: Springer; 1987.

[22] Rohan E. Existence and uniqueness of solutions of the discretized contact elasto-plastic problem with isotropic hardening. Math Models Meth Appl Sci 2000;10(08):1151-79.

[23] Rohan E. Sensitivity strategies in modelling heterogeneous media undergoing finite deformation. Math Comput Simul 2003;61(3-6):261-70.

[24] Rohan E, Lukeš V. Modeling nonlinear phenomena in deforming fluidsaturated porous media using homogenization and sensitivity analysis concepts. Appl Math Comput 2015;267:583-95.

[25] Rohan E, Whiteman JR. Shape optimization of elasto-plastic structures and continua. Comput Meth Appl Mech Eng 2000;187(1):261-88.

[26] Sanchez-Palencia E. Non-homogeneous media and vibration theory. Lecture notes in physics, vol. 127. Berlin: Springer; 1980.

[27] Temizer A, Wriggers P. A multiscale contact homogenization technique for the modeling of third bodies in the contact interface. Comput Meth Appl Mech Eng 2008;198(3):377-96. 